\title{
Dynamic Display of Changing Posterior in Bayesian Survival Analysis: The Software
}

\author{
Hani J. Doss \\ Department of Statistics \\ The Ohio State University \\ Columbus, $\mathrm{OH} 43210$ \\ B. Narasimhan \\ Department of Statistics \\ Stanford University \\ Stanford, CA 94305 \\ Revision : 1.34 of Date $: 1998 / 07 / 0217: 16: 33$
}

\section{Contents}

1 Copyright 4

2 Introduction $\quad 5$

3 The Software $\quad 5$

4 The Master Object Prototype $\quad 7$

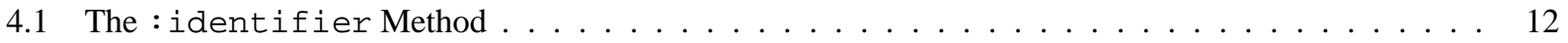

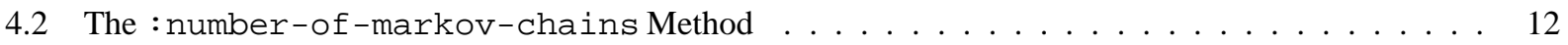

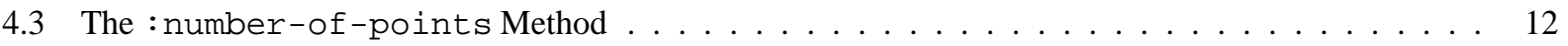

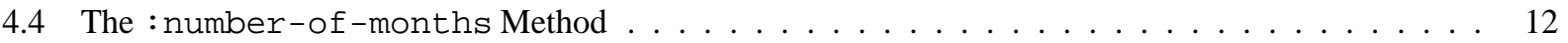

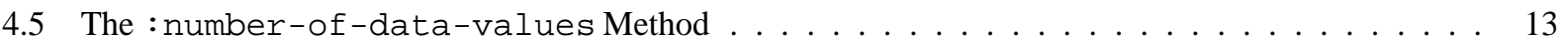

4.6 The $:$ summary-data Method . . . . . . . . . . . . . . . . . . . 13

4.7 The $:$ indicator-counts Method . . . . . . . . . . . . . . . . . 13

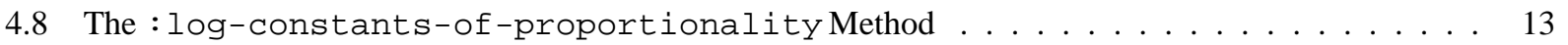

4.9 The $:$ slaves Method . . . . . . . . . . . . . . . . . . . . . . . . . . . . 14

4.10 The : initially-specified-hyperparameter-values Method . . . . . . . . . . 14

4.11 The $:$ hyperparameter-names Method . . . . . . . . . . . . . . . . . . . . 14

4.12 The : current-hyperparameter-values Method . . . . . . . . . . . . . . . 15

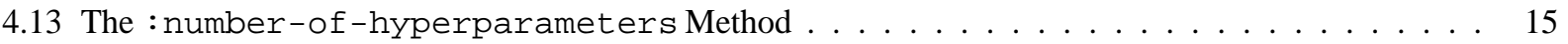

4.14 The :hyperparameters-used-in-markov-chains Method . . . . . . . . . . 16

4.15 The $:$ hyperparameter-ranges Method . . . . . . . . . . . . . . . . 16

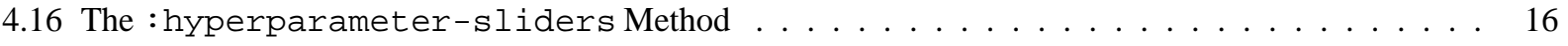

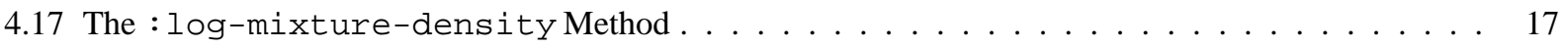

4.18 The :importance-weights Method . . . . . . . . . . . . . . . . . 17

4.19 The $: \log l i k$ Method . . . . . . . . . . . . . . . . . . . . . . . 17

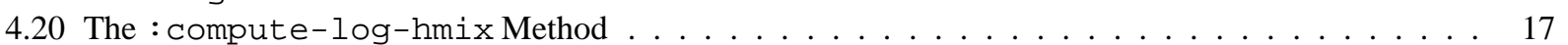

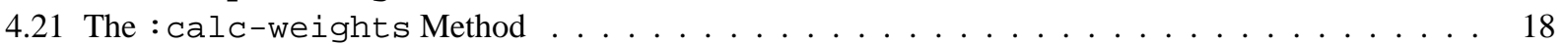

4.22 The $:$ isnew Method . . . . . . . . . . . . . . . . . . . . . 18

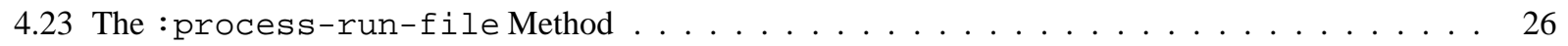

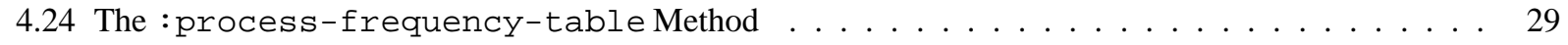

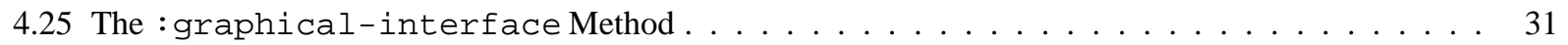

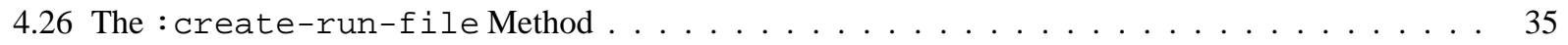


4.27 The $:$ synchronize Method . . . . . . . . . . . . . . . . . . . . 38

4.28 The : consolidate-computation Method .................... 39

4.29 The $:$ reset Method . . . . . . . . . . . . . . . . . . . . . . . . . . . . . . . . . 39

4.30 The $:$ effective-sample-size Method . . . . . . . . . . . . . . . . . . 39

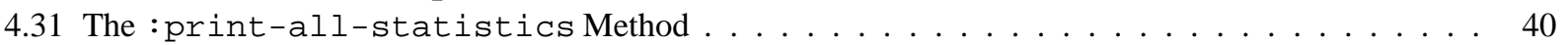

4.32 The : labelled-hyperparameter-values Method . . . . . . . . . . . . . 40

4.33 The $:$ statistics Method . . . . . . . . . . . . . . . . . . . . 41

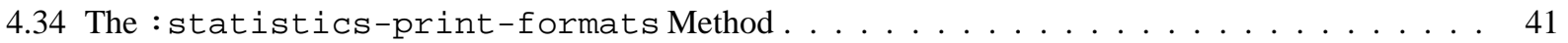

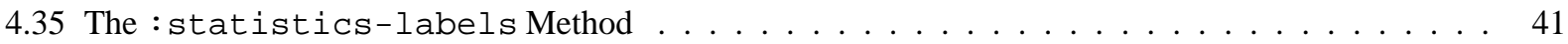

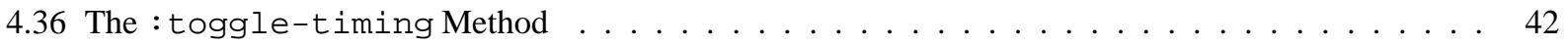

4.37 The $:$ superimpose Method . . . . . . . . . . . . . . . . . . . . 42

4.38 The $:$ close Method . . . . . . . . . . . . . . . . . . . . . 42

4.39 Defaults for Master . . . . . . . . . . . . . . . . . . . . . . . . . . 43

5 The Slave Object Prototype 43

5.1 The $:$ isnew Method . . . . . . . . . . . . . . . . . . . . . . 44

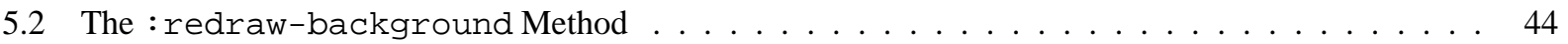

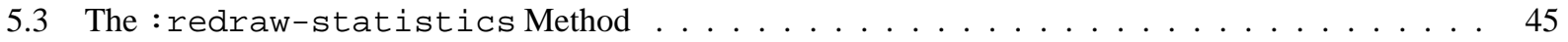

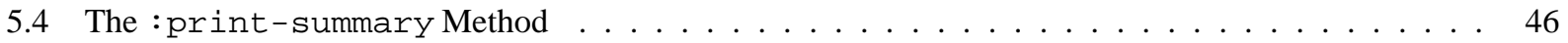

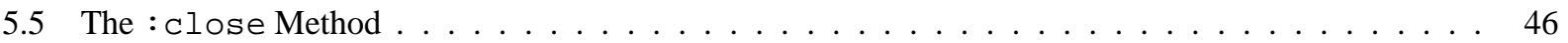

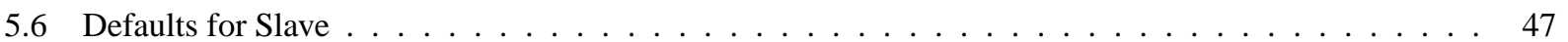

6 The C Programs 47

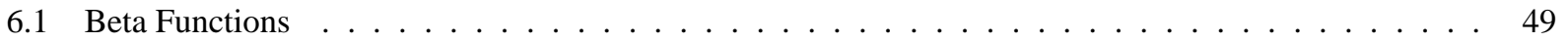

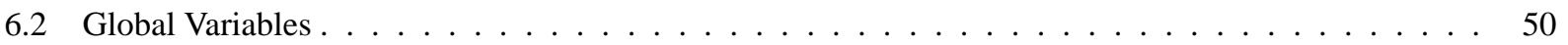

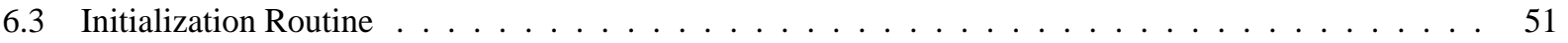

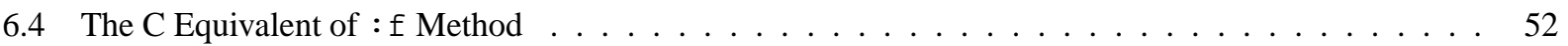

6.5 The $\mathrm{C}$ Equivalent of $: \operatorname{logp}$ Method $\ldots \ldots \ldots \ldots \ldots \ldots \ldots$

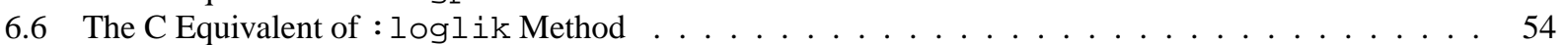

6.7 The C Equivalent of $:$ htheta-over-hmix Method . . . . . . . . . . . . . . . . 54

6.8 The C Equivalent of : compute-log-hmix Method . . . . . . . . . . . . . . . . 55

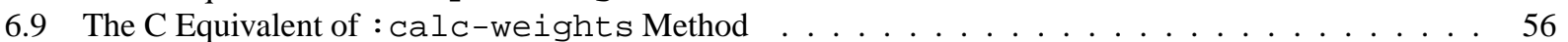

6.10 The C Equivalent of : compute-statistics Method . . . . . . . . . . . . . . . . . . 57

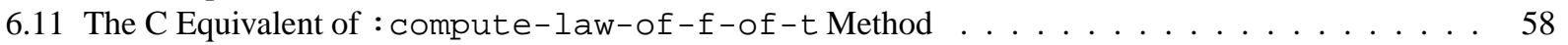

6.12 The $\mathrm{C}$ Equivalent of : compute-mean-of-fbar-of-t Method . . . . . . . . . . . . . 59

6.13 The $:$ consolidate-computation Method in C . . . . . . . . . . . . . 60

7 Installation Information $\quad 61$

$\begin{array}{llr}8 & \text { Improvements needed } & 67\end{array}$

9 Acknowledgement $\quad 67$

10 Index of Code Chunks $\quad 67$

11 Index of Identifiers $\quad 69$ 


\begin{abstract}
We consider the problem of estimating an unknown distribution function $F$ in the presence of censoring under the conditions that a parametric model is believed to hold approximately. We use a Bayesian approach, in which the prior on $F$ is a mixture of Dirichlet distributions. A hyperparameter of the prior determines the extent to which this prior concentrates its mass around the parametric family. A Gibbs sampling algorithm to estimate the posterior distributions of the parameters of interest is reviewed. An importance sampling scheme enables us to use the output of the Gibbs sampler to very quickly recalculate the posterior when we change the hyperparameters of the prior. The calculations can be done sufficiently fast to enable the dynamic display of the changing posterior as the prior hyperparameters are varied.
\end{abstract}

This paper provides a literate program completely documenting the code for performing the dynamic graphics. 


\section{Copyright}

We begin with our usual copyright.

$4\langle$ Copyright 4$\rangle \equiv$

$i$ i

; $;$; \$Revision: 1.34 \$ of \$Date: 1998/07/02 17:16:33 \$

$i ;$;

; ; $;$ Copyright (C) 1994, 1995, 1998. Doss and Narasimhan

i i i

; $;$; Hani J. Doss (dosslstat.ohio-state.edu) and

; $;$; B. Narasimhan (narasestat.stanford.edu)

; i ;

; $;$; This program is free software; you can redistribute it and/or modify

; $;$ it under the terms of the GNU General Public License as published by

; $;$; the Free Software Foundation; either version 2 of the License, or

; $;$; (at your option) any later version.

$i ; i$

; $;$; This program is distributed in the hope that it will be useful,

; $;$; but WITHOUT ANY WARRANTY; without even the implied warranty of

; $;$; MERCHANTABILITY or FITNESS FOR A PARTICULAR PURPOSE. See the

; $;$; GNU General Public License for more details.

$i ; i$

; $;$; You should have received a copy of the GNU General Public License

; ; ; along with this program; if not, write to the Free Software

; $;$; Foundation, Inc., 675 Mass Ave, Cambridge, MA 02139, USA.

$i ;$;

Defines:

copyright, never used. 


\section{Introduction}

This document is a literate program implementing the theory described in our paper [2]. A literate program is a program written in a style that makes it easy for humans to read, understand and modify. For more information on Literate Programming, see [4]. A quicker introduction is available on the World Wide Web; see [6]. This document uses the Noweb [7], [8], [9] literate programming tools. Although it is not required, we recommend that any serious user of this software have the Noweb tools installed. Noweb tools, besides being free, are extremely easy to install and require no special expertise other than basic knowledge about how $\mathrm{T}_{\mathrm{E}} \mathrm{X}$ or $\mathrm{LT}_{\mathrm{E}} \mathrm{X}$ work. Having Noweb allows one to take full advantage of our software-syntax errors will be minimized and the code we have written can be reused with the user's modifications spliced into place automatically by Noweb.

We expect any serious user of our software to read the original paper [2], a copy of which is included in the software distribution.

This document is available in three forms: Postscript, PDF and HTML. All versions are accessible from the web pages of the authors.

We wish to remark that the software only does sensitivity analysis. No general facility is provided for generating observations from Markov chains. Indeed, since the range of models for which MCMC methods are applicable is large and such methods most likely involve problem-specific issues, it is our opinion that building such a supertool, if it is at all possible, is a non-trivial task. However, the Fortran program used in generating the output for our example is included along with this software and can be used for models similar to ours. Of course, any appropriate method may be used to generate the samples as long as the output is available in a form usable by our software. The requirements on the data that can be used with our software are spelt out below.

Corresponding to each Markov chain output, there must be two files with the extensions . in (input file) and . out (output file). For example, mc1. in and mc1. out.

The input file must have the following structure. The first four items in the file can be anything, string or number, either on a single line or any conceivable combination of lines. The next three items must be the shape of the Gamma distribution on $\theta$, the scale of the Gamma distribution on $\theta$-the parametrization for shape $a$ and scale $b$ is proportional to $\theta^{a-1} \exp (-b \theta)$ - and $\alpha(\mathbf{R})$. The next three values values following these quantities can be anything, but the one following it should be the number of data points, or sets. Nothing else is read from the input file.

The output file must have the following structure for each data point generated by the Markov chain. The value of $\theta$ must be followed by the number of distinct values of the data points, which must be followed by a frequency table of the actual data value and the corresponding frequency. The layout of the values on lines does not matter as long as at least a single white space delimits values. If this structure is violated, errors will result. A peek at the data files included with this software will help the reader.

A note on performance. The calculations involved in reweighting are non-trivial and require a reasonably powerful computer for smooth performance. The efficiency can be improved by dynamically loading $\mathrm{C}$ programs that compute various quantities. The version of software described here does so by default. An older version that does not use dynamic loading which is available upon request from the authors. Without dynamic loading, the performance is very bad indeed.

Dynamic libraries for Windows and Macintosh are provided. Suggestions for various Unix platforms are also provided. Section 7 has more details.

It is assumed that a proper installation of Lisp-Stat described in [10] is available. The version number on Lisp-Stat should be 3.52.0 or higher since dynamic loading uses the new shared library mechanism.

Some additions planned for the future are listed in Section 8.

\section{The Software}

The software consists of the following components.

$5\langle * 5\rangle \equiv$

$\langle$ Copyright 4$\rangle$

(require "utility") 


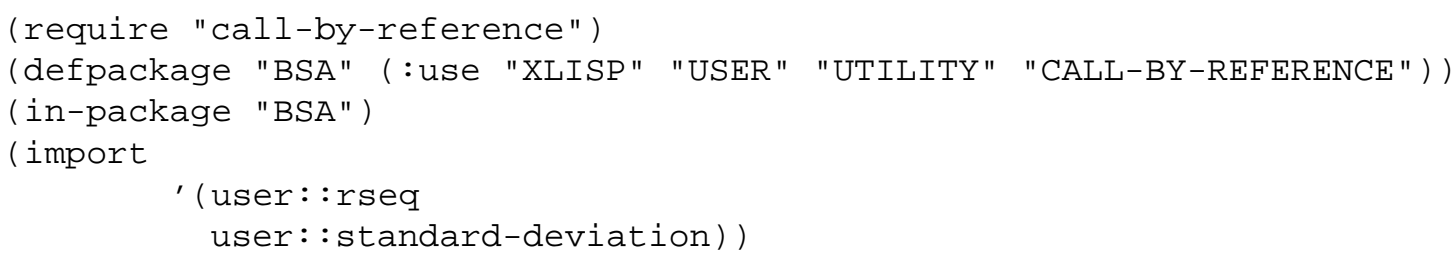




\section{The Master Object Prototype}

The master prototype master-proto inherits from dialog-proto of Lisp-Stat and contains a number of slots. A rich set of methods facilitate interaction with the master.

' (identifier number-of-markov-chains

number-of-points

number-of-data-values

number-of-months

data-file-names

summary-data

indicator-counts

log-constants-of-proportionality

slaves

hyperparameters-used-in-markov-chains

initially-specified-hyperparameter-values

current-hyperparameter-values

hyperparameter-names

log-mixture-density

importance-weights

hyperparameter-ranges

hyperparameter-sliders

work-space

shared-library

density-abscissae

density-ordinates

expectation-abscissae

expectation-ordinates

standard-deviation-ordinates

statistics

statistics-print-formats

statistics-labels

number-of-slider-stops

superimpose

timing

timing-button

lazy)

() dialog-proto

"The Master prototype. Creates and manipulates a harem of slaves.")

$\langle$ Methods for Master Prototype 10〉

$\langle$ Defaults for Master 43a〉

Defines:

current-hyperparameter-values, used in chunks 15a, 25, 38-40, 46a, and 61.

data-file-names, never used.

density-abscissae, never used.

density-ordinates, never used

expectation-abscissae, never used.

expectation-ordinates, never used.

hyperparameter-names, used in chunks 14c, 24b, and 40a.

hyperparameter-ranges, used in chunks 16b, 20, 24b, 25a, 27d, and 36b.

hyperparameter-sliders, used in chunk 16c.

hyperparameters-used-in-markov-chains, used in chunk 16a. 


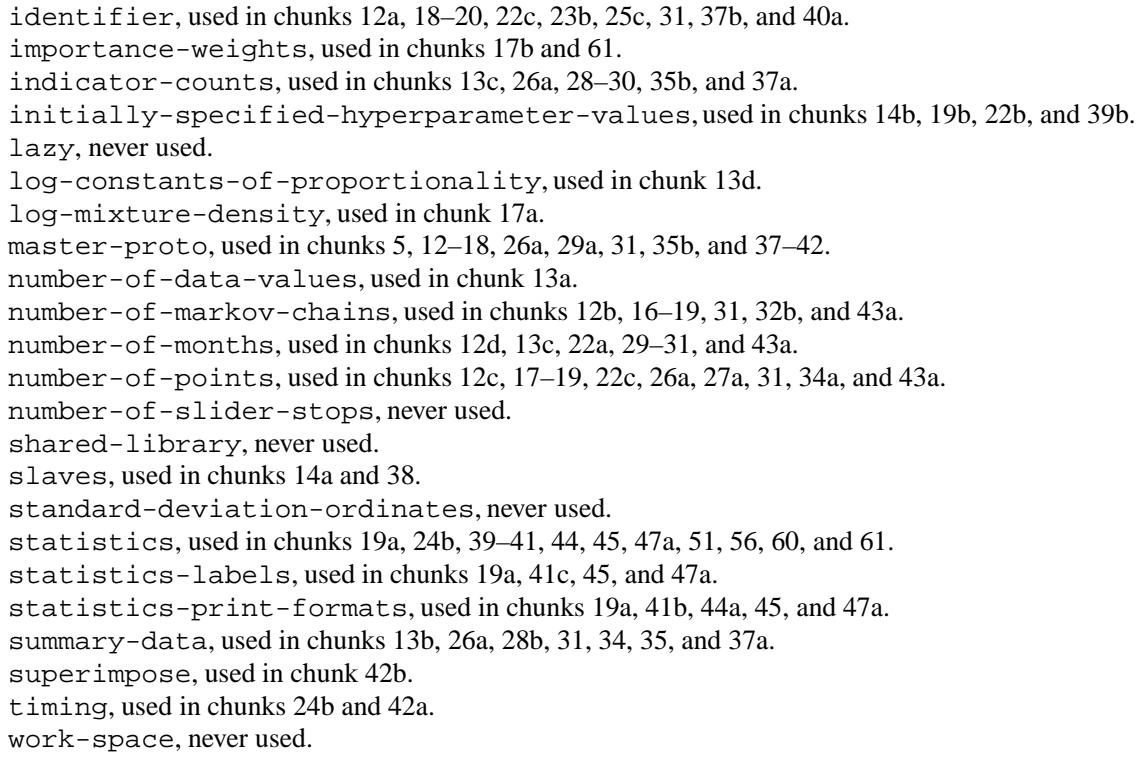


Throughout, we shall use $m$ for the number of Markov chains, and $n$ for the number of data points in the output of each Markov chain. Note that arrays are indexed from 0 so that index $i$ refers to the $(i+1)$-th position.

Here is a description of all the slots in master-proto.

identifier holds a string that is used to identify instances of the object. This string also helps in repeating any interesting run-all inputs needed for the run are saved in a file whose name is generated by adding an extension . run to the string. In addition, a file with the extension . lsp is also created so that invoking $x l$ ispstat on that file with automatically recreate everything without annoying questions.

number-of-markov-chains holds the number of Markov chains $(m)$ to be used in the exploration.

number-of-points holds the number of data points from each Markov chain $(n)$ to be used in the exploration.

number-of-data-values holds the number of data values, that is the number of sets.

number-of-months holds the number of months for which the law of $F(t)$ is computed. Default is given by the variable *default-number-of-months*.

data-file-names holds a list of the data files corresponding to the Markov chains, without the extension. Dimension is $m$.

summary-data This slot holds the summary data from all the Markov chains. The data is stored as a 2-d array with each row holding $\left(d_{x}, \theta\right)$, where $d_{x}$ is the the number of distinct values of the data points. The first set of $n$ pairs correspond to the first Markov chain, the next set to the second and so on, which means that the indices have to be suitably translated to access values. There are $m n$ pairs in all. Note that we only handle balanced data, i.e., same $n$ for all Markov chains.

indicator-counts This slot is a 2-d array of size $m n \times 2$ containing the a count of the number of datavalues less than $t$, the number of months. The number of columns in this array is indicated by the slot number-of-months. If this value is 61 , say, then one would be able to calculate the law of $F(t)$ for $t$ ranging from 0 to 60 months.

log-constants-of-proportionality holds a vector of estimates of the logs of the constants of proportionality, $\left(\tilde{C}\left(\kappa_{i}\right)\right)^{-1}$, in the paper. The first constant is implicitly -1 and so the dimension of this slot is $m-1$.

slaves stores a list of slaves who need to be informed of changes in the hyperparameter values. Each slave is an instance of slave-proto.

hyperparameters-used-in-markov-chains stores an $m$ by 3 array holding the values of hyperparameters at which the Markov chains were run. If $a$ is the array by $a$, then $a[j, i]$ is the value of the $i$-th hyperparameter for the $j$-th Markov chain, $0 \leq j<m$ and $0 \leq i<3$. The three hyperparameters in every row are $\alpha(\mathbf{R})$, the shape and scale of the Gamma prior on $\theta$ respectively.

initially-specified-hyperparameter-values is a $4 \times 1$ array of initial values for the hyper-parameters at which the exploration should begin. These values serve are used in starting and restarting the exploration. The one extra hyperparameter is the value of time $t$ in $F(t)$.

current-hyperparameter-values is a $4 \times 1$ array of the current values of the three hyperparameters. These are the quantities that are changed by the user via sliders.

hyperparameter-names holds a list of names for the hyper-parameters. Default is Alpha, Theta Shape, Theta Scale, and Time (Months).

log-mixture-density is a an array holding $\log h_{m i x}(x)$ at the data points. Size is $m n$. See [3]. For speed in dynamic graphics, this quantity is calculated once and saved. 
importance-weights holds the importance weights $w[i], 0 \leq i<n m$, used for the Reweighting Mixtures (RM) scheme. See [1] and [3].

hyperparameter-ranges stores a list of hyperparameter ranges to be used in exploration. For internal use only.

hyperparameter-sliders is a list of slider objects for internal use only.

work-space is a slot used for storing results from dynamically loaded $\mathrm{C}$ routines.

shared-library holds a library for shared handle when dynamic loading is used. For internal use only.

density-abscissae is a vector of abscissa values between 0 and 1 at which the density of $F(t)$ will be plotted. This is an array of length *default-number-of-plot-stops*.

density-ordinates is a vector of values of the density of $F(t)$. This is an array of length

*default-number-of-plot-stops*.

expectation-abscissae is a vector of values of $E(\bar{F}(t))$ for $t$ ranging from 0 to

*default-number-of-months* (minus 1 , as we start at 0 ).

expectation-ordinates is a vector of values of $E(\bar{F}(t))$ for $t$ ranging from 0 to

*default-number-of-months* (minus 1 , as we start at 0 ).

standard-deviation-ordinates is a vector of values of $\sigma_{\bar{F}(t)}$.

statistics is a vector of 3 values that will hold $E(\bar{F}(t)), \sigma_{\bar{F}(t)}$ and the effective sample size. The effective sample size is calculated using the formula

$$
\text { Effective Sample Size }=m * n /\left(1+c v(W)^{2}\right),
$$

where $c v(W)$ is the coefficient of variation of the importance weights $W$. See, for example, [5].

statistics-print-formats is a list of lists indicating the format to be used in printing the statistics values.

statistics-labels is a list of strings (labels).

number-of-slider-stops stores the number of slider stops for hyperparameters.

superimpose toggles superimposition on and off. Default is off.

timing signifies if timing is needed.

timing-button is a button for toggling timing on and off.

lazy is a slot used for efficient synchronization. It is for internal use by programs and the user shouldn't mess with it.

The methods for master-proto can be broken down as follows. 
July 2, 1998

bsa.nw

$\langle$ The Master :identifier Method 12a

$\langle$ The Master :number-of-markov-chains Method 12b〉

$\langle$ The Master : number-of-points Method 12c〉

$\langle$ The Master :number-of-months Method 12d

〈The Master : number-of-data-values Method 13a〉

$\langle$ The Master :summary-data Method 13b

$\langle$ The Master :indicator-counts Method 13c

$\langle$ The Master :log-constants-of-proportionality Method 13d〉

〈The Master :slaves Method 14a〉

〈The Master :initially-specified-hyperparameter-values Method 14b

〈The Master :hyperparameter-names Method 14c〉

〈The Master :current-hyperparameter-values Method 15a)

〈The Master : number-of-hyperparameters Method 15b〉

〈The Master :hyperparameters-used-in-markov-chains Method 16a〉

〈The Master :hyperparameter-ranges Method 16b〉

〈The Master :hyperparameter-sliders Method 16c〉

$\langle$ The Master :log-mixture-density Method 17a)

$\langle$ The Master :importance-weights Method 17b

$\langle$ The Master :loglik Method 17c)

$\langle$ The Master :compute-log-hmix Method 17d

$\langle$ The Master :calc-weights Method 18a)

〈The Master :isnew Method 18b

〈The Master : graphical-interface Method 31〉

〈The Master :process-run-file Method 26a)

〈The Master :process-frequency-table Method 29a)

$\langle$ The Master :create-run-file Method 35b

〈The Master :synchronize Method 38〉

$\langle$ The Master :consolidate-computation Method 39a)

〈The Master :reset Method 39b

$\langle$ The Master :effective-sample-size Method 39c〉

$\langle$ The Master :print-all-statistics Method 40a〉

〈The Master :labelled-hyperparameter-values Method 40b〉

$\langle$ The Master :statistics Method 41a〉

$\langle$ The Master :statistics-print-formats Method 41b

〈The Master :statistics-labels Method 41c〉

$\langle$ The Master : superimpose Method 42b〉

$\langle$ The Master :toggle-timing Method 42a)

$\langle$ The Master :close Method 42c〉 
July 2, 1998

Some of these methods are mere accessor and modifier methods for the slots and we can get them easily out of the way.

\subsection{The : identifier Method}

$\langle$ The Master :identifier Method 12a $\equiv$

(defmeth master-proto :identifier (\&optional name)

"Method args: (\&optional name)

Sets or retrieves the identifier slot."

(if name

(setf (slot-value 'identifier) name)

(slot-value 'identifier)))

Defines:

: identifier, used in chunks 19a, 25c, 37b, and 40a.

Uses identifier 7 and master-proto 7.

\subsection{The : number-of-markov-chains Method}

$\langle$ The Master :number-of-markov-chains Method 12b〉三

(defmeth master-proto : number-of-markov-chains ()

"Method args: ()

Returns $m$, the number of Markov chains used."

(slot-value 'number-of-markov-chains))

Defines:

: number-of-markov-chains, used in chunk 19a.

Uses master-proto 7 and number-of-markov-chains 7.

\subsection{The : number-of-points Method}

12c $\langle$ The Master :number-of-points Method 12c $\rangle \equiv$

(defmeth master-proto : number-of-points ()

"Method args: ()

Returns $n$, the number of data points."

(slot-value 'number-of-points))

Defines:

: number-of-points, used in chunk 19a.

Uses master-proto 7 and number-of-points 7.

\subsection{The : number-of-months Method}

$\langle$ The Master :number-of-months Method 12d〉三

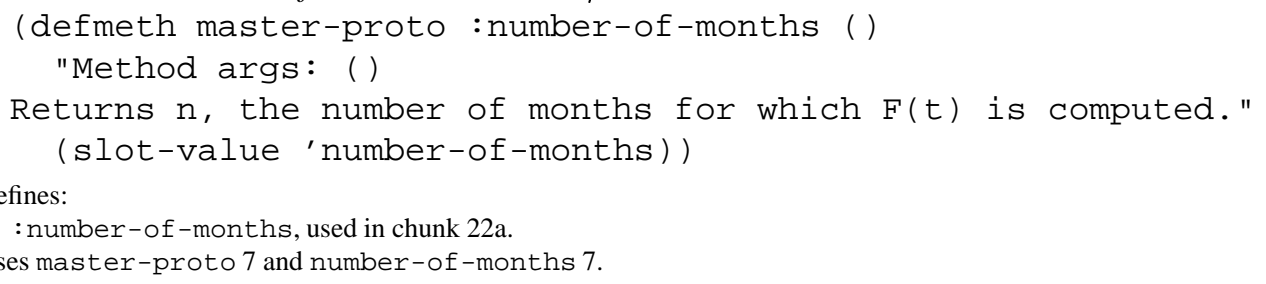


July 2, 1998

\subsection{The : number-of-data-values Method}

13a 〈The Master :number-of-data-values Method 13a〉三

(defmeth master-proto : number-of-data-values ()

"Method args: ()

Returns $n$, the number of data values, or sets."

(slot-value 'number-of-data-values))

Defines:

: number-of-data-values, never used.

Uses master-proto 7 and number-of-data-values 7.

\subsection{The : summary-data Method}

13b $\quad\langle$ The Master : summary-data Method 13b $\rangle$

(defmeth master-proto : summary-data ()

"Method args: ()

Returns the summary data containing pairs theta and number of distinct data points. A 2 d array of size mn by $2 . "$

(slot-value' summary-data))

Defines:

: summary-data, never used.

Uses master-proto 7 and summary-data 7.

\subsection{The : indicator-counts Method}

13c $\quad\langle$ The Master :indicator-counts Method 13c〉三

(defmeth master-proto : indicator-counts ()

"Method args: ()

Returns the data values a 2 d array of size mn by number-of-months."

(slot-value' indicator-counts))

Defines:

: indicator-counts, never used.

Uses indicator-counts 7, master-proto 7, and number-of-months 7.

\subsection{The : log-constants-of-proportionality Method}

13d 〈The Master :log-constants-of-proportionality Method 13d $\rangle \equiv$

(defmeth master-proto :log-constants-of-proportionality ()

"Method args: ()

Returns a list of values of the log constants of

proportionality for the posteriors for each Markov chain."

(slot-value' 'log-constants-of-proportionality))

Defines:

: log-constants-of-proportionality, never used.

Uses log-constants-of-proportionality 7 and master-proto 7. 


\subsection{The : slaves Method}

"Method args: () Retrieves the slaves."

(slot-value'slaves))

Defines:

: slaves, never used.

Uses master-proto 7 and slaves 7 .

\subsection{The : initially-specified-hyperparameter-values Method}

$\langle$ The Master :initially-specified-hyperparameter-values Method 14b〉三

(defmeth master-proto : initially-specified-hyperparameter-values ()

"Method args: ()

Returns a list of initially specified values of the hyper

parameters. Used mainly for resetting the hyper parameter values

before dynamic exploration."

(slot-value 'initially-specified-hyperparameter-values))

Defines:

:initially-specified-hyperparameter-values, used in chunks 19b, 22b, and 39b.

Uses initially-specified-hyperparameter-values 7 and master-proto 7.

\subsection{The : hyperparameter-names Method}

$\langle$ The Master :hyperparameter-names Method 14c $\rangle$

(defmeth master-proto :hyperparameter-names (\&optional index)

"Method args: (index)

Returns a string identifying the hyperparameter index or the whole slot." (if index

(select (slot-value 'hyperparameter-names) index)

(slot-value 'hyperparameter-names)))

Defines:

: hyperparameter-names, used in chunk $24 \mathrm{~b}$.

Uses hyperparameter-names 7 and master-proto 7. 
July 2, 1998

\subsection{The : current-hyperparameter-values Method}

"Method args: (\&optional i $\mathrm{x}$ )

Returns a list of hyper-parameter values. If $i$ is given and a sequence, then all values in the list are set. The dimensions must match. If i is not a sequence, returns the i-th value. If $x$ is specified, sets the $i-t h$ hyperparameter value to $x$. Note that $x$ is ignored if $i$ is a sequence." (if $x$

(if (slot-value' 'timing)

(time

(progn

(setf (elt (slot-value 'current-hyperparameter-values) i) $x$ )

(send self : synchronize)))

(progn

(setf (elt (slot-value 'current-hyperparameter-values) i) x)

(send self : synchronize)))

(if $i$

(if (sequencep i)

(progn

(setf (slot-value 'lazy) t)

(dotimes ( $j$ (send self : number-of-hyperparameters))

(send (select (slot-value 'hyperparameter-sliders) j) : value (select $i j))$ )

(setf (slot-value' lazy) nil)

(send self : synchronize))

(elt (slot-value 'current-hyperparameter-values) i))

(slot-value 'current-hyperparameter-values))))

Defines:

: current-hyperparameter-values, used in chunks 25, 38-40, 46a, and 61.

Uses current-hyperparameter-values 7, master-proto 7, :number-of-hyperparameters $15 \mathrm{~b}$, and : synchronize 38.

\subsection{The : number-of-hyperparameters Method}

Defines:

: number-of-hyperparameters, used in chunks 15a, 25, and 40b.

Uses master-proto 7. 
July 2, 1998

bsa.nw

\subsection{The :hyperparameters-used-in-markov-chains Method}

$16 \mathrm{a}$

$\langle$ The Master :hyperparameters-used-in-markov-chains Method 16a $\rangle \equiv$ (defmeth master-proto :hyperparameters-used-in-markov-chains ()

"Method args: ()

Returns the hyperparameter-values used in running the Markov

chains. 2D array of size number-of-markov-chains by 3."

(slot-value 'hyperparameters-used-in-markov-chains))

Defines:

: hyperparameters-used-in-markov-chains, never used.

Uses hyperparameters-used-in-markov-chains 7, master-proto 7, and number-of-markov-chains 7.

\subsection{The : hyperparameter-ranges Method}

$16 b$

$\langle$ The Master :hyperparameter-ranges Method 16b $\equiv$

(10)

(defmeth master-proto : hyperparameter-ranges (\&optional ranges)

"Method args: (\&optional ranges)

Returns the ranges within which the hyperparameters will be

varied. List of lists. This is calculated as the maximum and minimum

of all the values used in the Markov chains if not given."

(if ranges

(setf (slot-value 'hyperparameter-ranges) ranges)

(if (slot-value' hyperparameter-ranges)

(slot-value 'hyperparameter-ranges)

(let* ( values (column-list

(slot-value 'hyperparameters-used-in-markov-chains)))

(max (mapcar \#'max values))

(min (mapcar \#'min values)))

(append (mapcar \#' (lambda ( $\mathrm{x} y)$ (list $\mathrm{x} y$ )) $\min \max$ )

(list (list 0 (1-(slot-value 'number-of-months)))))))))

Defines:

: hyperparameter-ranges, used in chunks 20, 24b, 27d, and 36b.

Uses hyperparameter-ranges 7 and master-proto 7.

\subsection{The :hyperparameter-sliders Method}

$16 \mathrm{c}$

$\langle$ The Master :hyperparameter-sliders Method 16c $\rangle \equiv$

(defmeth master-proto :hyperparameter-sliders ()

"Method args: ()

Returns the sliders associated with the hyperparameters."

(slot-value 'hyperparameter-sliders))

Defines:

: hyperparameter-sliders, never used.

Uses hyperparameter-sliders 7 and master-proto 7. 
July 2, 1998

$\mathrm{bsa} \cdot \mathrm{nw}$

\subsection{The : log-mixture-density Method}

17a 〈The Master :log-mixture-density Method 17a〉三

(defmeth master-proto :log-mixture-density ()

"Method args: ()

Returns the log of the mixture density at each of the data points.

An array of nm values."

(slot-value ' log-mixture-density))

Defines:

: log-mixture-density, never used.

Uses log-mixture-density 7 and master-proto 7.

\subsection{The : importance-weights Method}

$17 \mathrm{~b}$

$\langle$ The Master :importance-weights Method 17b $\equiv$

(defmeth master-proto :importance-weights ()

"Method args: ()

Retrieves the importance sampling weights. An array

of size number-of-markov-chains by number-of-points."

(slot-value' importance-weights))

Defines:

: importance-weights, never used.

Uses importance-weights 7, master-proto 7, number-of-markov-chains 7, and number-of-points 7.

\subsection{The : loglik Method}

17c $\langle$ The Master :loglik Method 17c $\rangle \equiv$

(defmeth master-proto :loglik (n)

"Returns the log-quasi-likelihood as a function of $\mathrm{n}$. The dimension

of $\mathrm{n}$ should be one less than the number of Markov chains."

(let ((result (slot-value 'work-space)))

(call-by-reference-oldcfun "logLikelihood"

(slot-value 'shared-library)

(coerce $\mathrm{n}^{\prime}$ (vector $\mathrm{c}^{- \text {double)) result) }}$

(aref result 0)) )

Defines:

: $\log l i k$, used in chunk $23 \mathrm{c}$.

Uses loglik 54a and master-proto 7.

\subsection{The : compute-log-hmix Method}

$\langle$ The Master :compute-log-hmix Method 17d $\rangle \equiv$

(10)

(defmeth master-proto :compute-log-hmix ()

"Method args: ()

Computes the log of the mixture density at the various data points."

(call-by-reference-oldcfun "computeLogHmix"

(slot-value' 'shared-library)))

Defines:

compute-log-hmix, used in chunk $23 \mathrm{~b}$.

Uses computeLogHmix 55 and master-proto 7. 
July 2, 1998

\subsection{The :calc-weights Method}

18a $\langle$ The Master :calc-weights Method 18a $\equiv$

(defmeth master-proto :calc-weights ()

"Method args: ()

Calculates the importance weights."

(call-by-reference-oldcfun "calcWeights" (slot-value 'shared-library))) Defines:

: calc-weights, used in chunk $18 \mathrm{~b}$.

Uses calcWeights 56 and master-proto 7.

\subsection{The : isnew Method}

The : isnew method for master-proto is a bit involved.

$\langle$ Set up slot values for master object $18 \mathrm{c}\rangle$

(send self :calc-weights)

$\langle$ Create slaves of master object 24a

$\langle$ Set up dialog and wait for user input 24b

$\langle$ Some final touches 25c $\rangle$ )

Defines:

: isnew, used in chunk 51.

Uses : calc-weights 18a, identifier 7, master-proto 7, number-of-markov-chains 7, and number-of-points 7.

The : synchronize message at the end of this method forces the computations anyway, so we avoid the computations now. In other words, initially, we want to be lazy in performing intensive computations. 
We need to determine $m$, the number of chains and $n$, the number of data points to be used. If the argument identifier? is given, then all the necessary inputs can be read from the "run" file. Otherwise, we need to prompt the user for everything.

Uses *default-shared-lib-name 43a, *default-statistics-labels*47a, *default-statistics-print-formats*47a, : identifier 12a, identifier 7, : number-of-markov-chains 12b, number-of-markov-chains 7 , : number-of-points 12c, number-of-points 7, :process-run-file 28b, statistics 7 50, statistics-labels 7 , and statistics-print-formats 7.

Time to get information on how the exploration is to proceed.

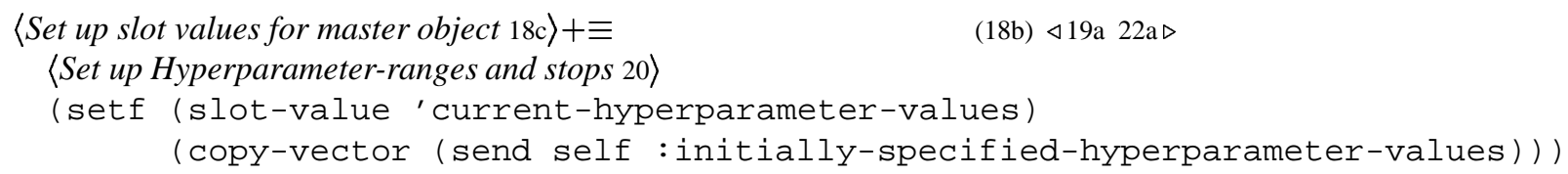


This code chunk sets up a large dialog box for all the various quantities. In most cases, the user will just continue without change.

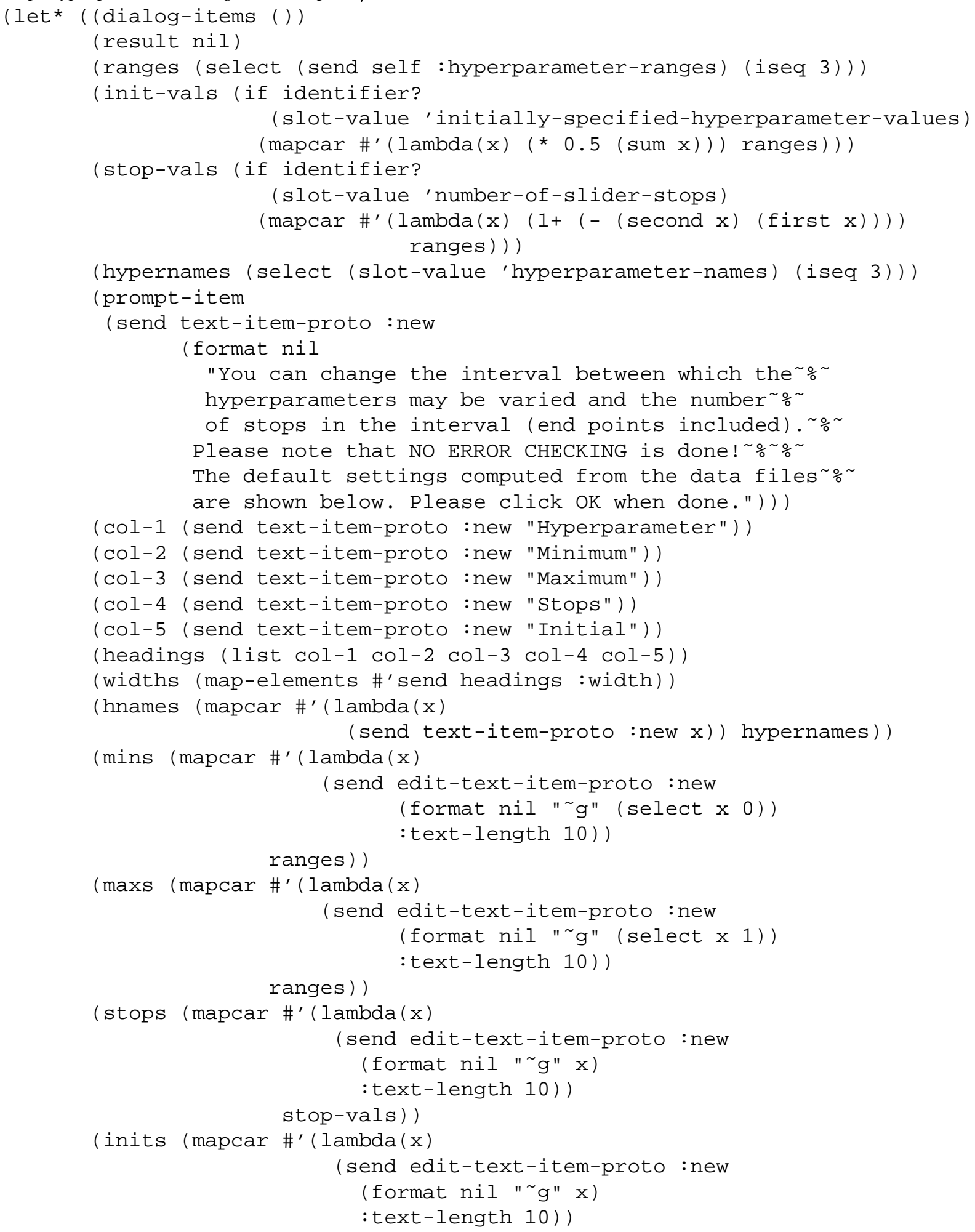




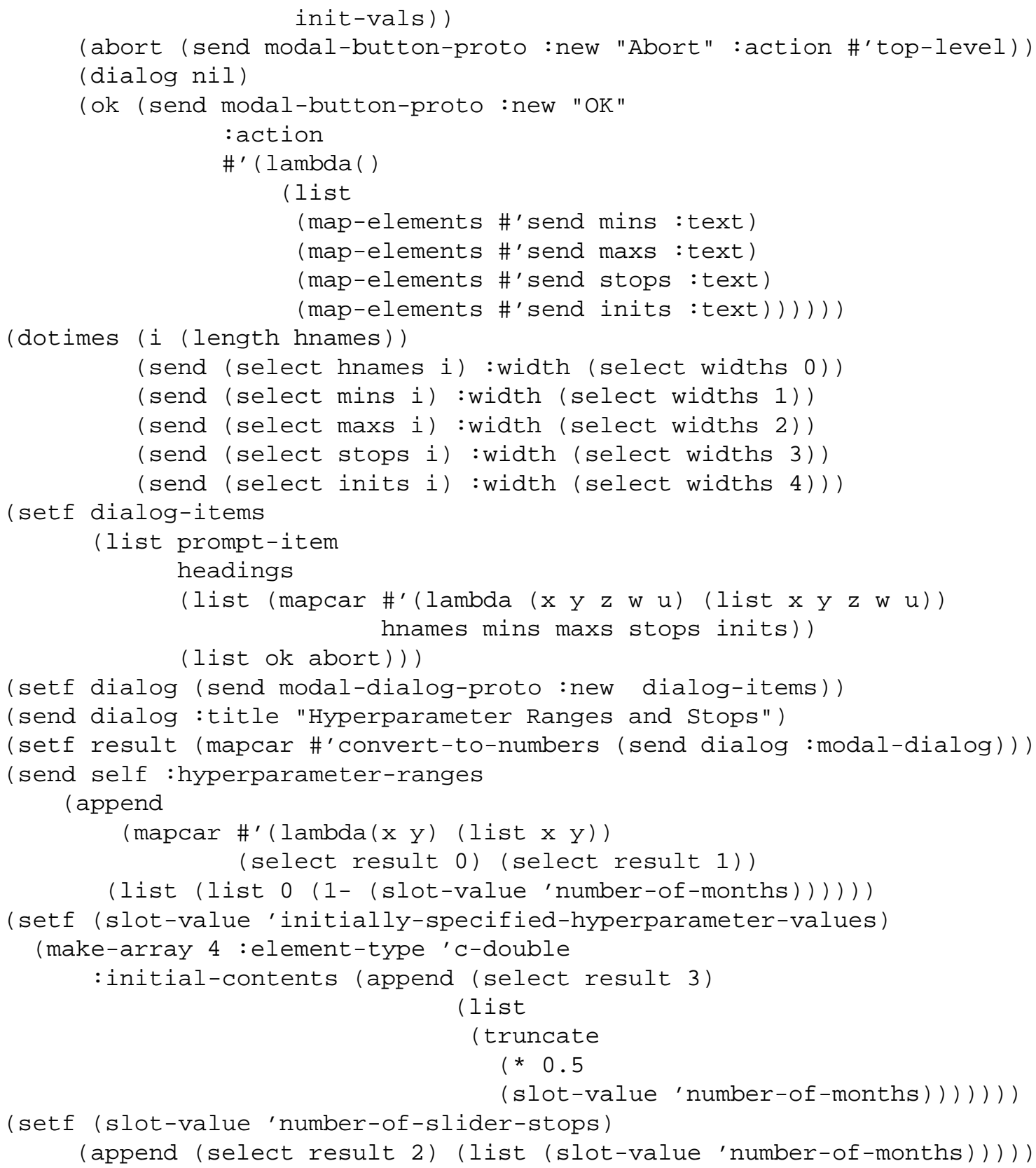


Now, make the initial arrays for calling the $\mathrm{C}$ functions.

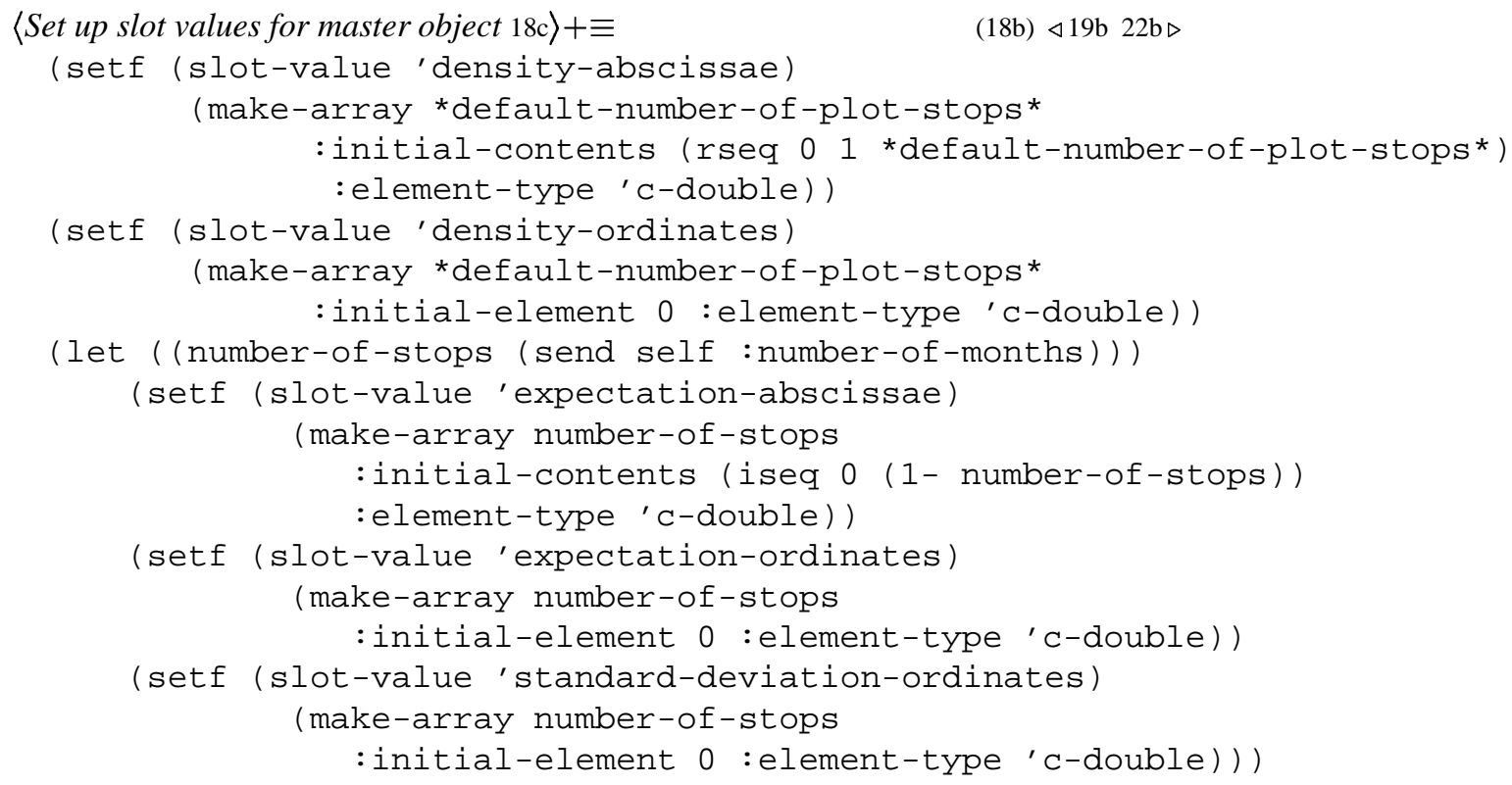

Uses : number-of-months $12 \mathrm{~d}$ and number-of-months 7.

The current values of the hyperparameters must be those specified initially.

(let ((tmp-array (send self : initially-specified-hyperparameter-values))) (setf (slot-value 'current-hyperparameter-values)

Uses : initially-specified-hyperparameter-values $14 \mathrm{~b}$ and initially-specified-hyperparameter-values 7.

We set up the number of points to be used in Reverse Logistic Regression if necessary. After that, all the data areas will have been set up and so we pass addresses of the data-arrays to the $\mathrm{C}$ routines.

$\langle$ Set up slot values for master object $18 \mathrm{c}\rangle+\equiv$

(18b) $\triangleleft 22 \mathrm{~b} 23 \mathrm{~b} \triangleright$

(let* ( ( (slot-value ' number-of-points) )

(nc (min *default-number-of-points* n)) )

(unless (or log-constants? identifier?

(= (slot-value 'number-of-markov-chains) 1))

(setf $\mathrm{nc}$

(select (get-tested-value-dialog

(format nil "How many points should I use from each chain for \% estimating the constants of proportionality? ${ }^{\circ}$ -

Unless you are prepared to wait for a long time, you \% should go with the default or less! \%")

:initial nc

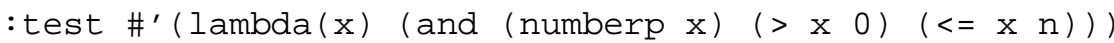

:error-message "Invalid entry. Please try again!")

0)))

(Pass data array addresses to C Routines 23a) )

Uses *default-number-of-points * 43a, identifier 7 , and number-of-points 7. 
We are ready to create the slave objects, a plot of the density of $F(t)$ as well as a plot of $E(S(t))$ for various values of $t$.

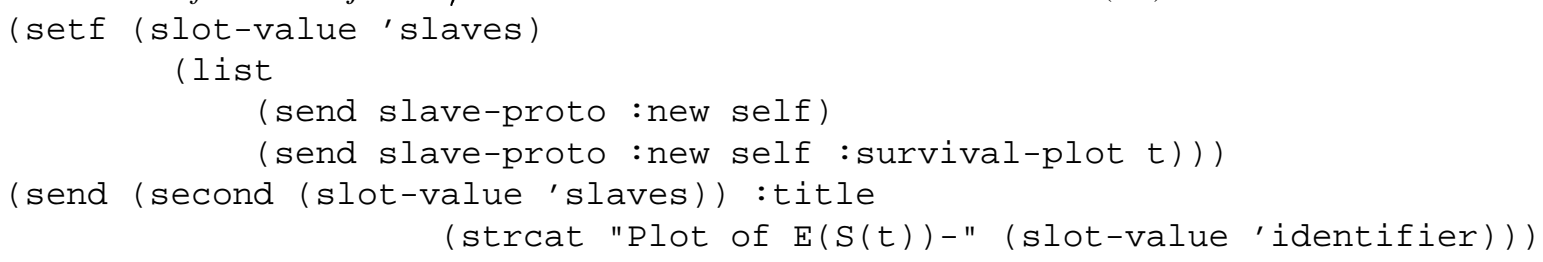

Uses slave-proto $43 b$.

The sliders for controlling the hyperparameters need to be set up. There are three dialog items that correspond to every hyperparameter: a text-item where the hyperparameter name will be displayed, a value-text-item where the value of the hyperparameter will be displayed and underneath the first two, a slider showing the slider stop. Figure 1 shows a typical slider. For nice looks, the width of the hyperparameter name string and the value string should add up to the width of the slider.

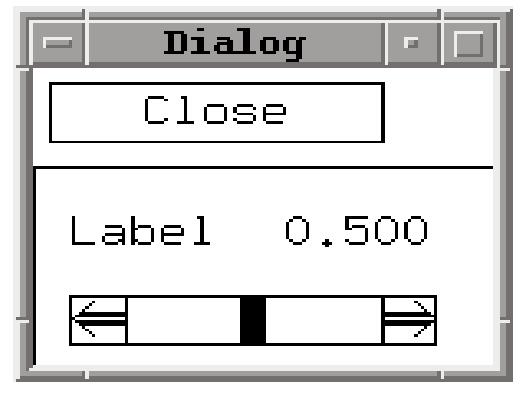

Figure 1: A typical slider in a dialog

Uses : hyperparameter-names 14c, hyperparameter-names 7, : hyperparameter-ranges 16b, hyperparameter-ranges 7 , :print-all-statistics 40a, : reset 39b, statistics 7 50, timing 7, and :toggle-timing 42a. 
Let us first create the triples we need for the hyperparameter sliders for use with the function make-sliders. The triples are (a) the label for the hyperparameter, (b) the range within which the hyperparameter will be varied, and (c) the action that is to be taken when the slider is pressed as a function.

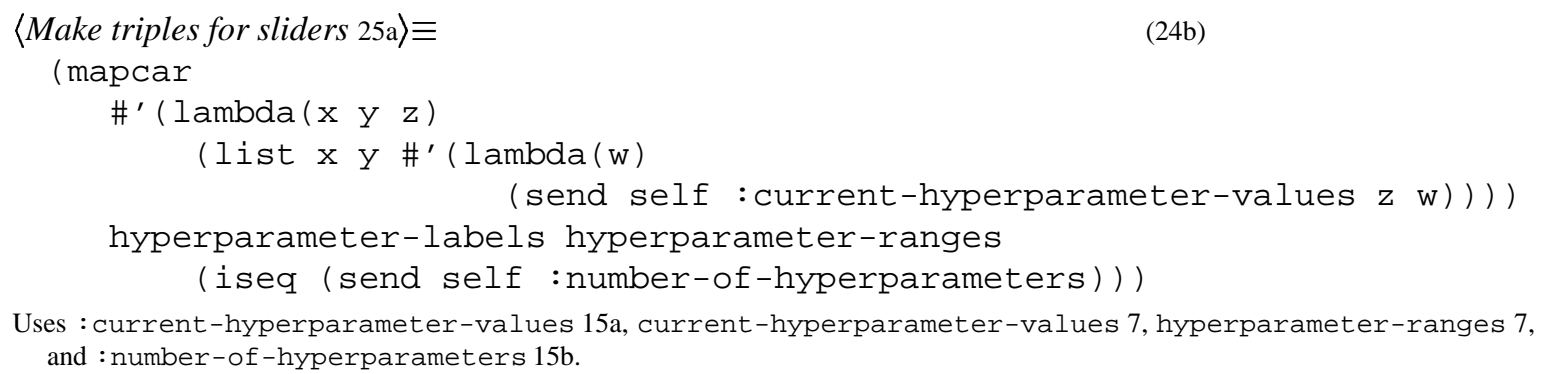

We are now ready to create the sliders and associated text and value items. The function make-sliders returns a multiple-values list of slider-items using triples with a specified layout along with a list of the appropriate scroll-items. A slider-item is a list of two elements, the first element being a list containing a text-item and a value-item and the second is a scroll-item.

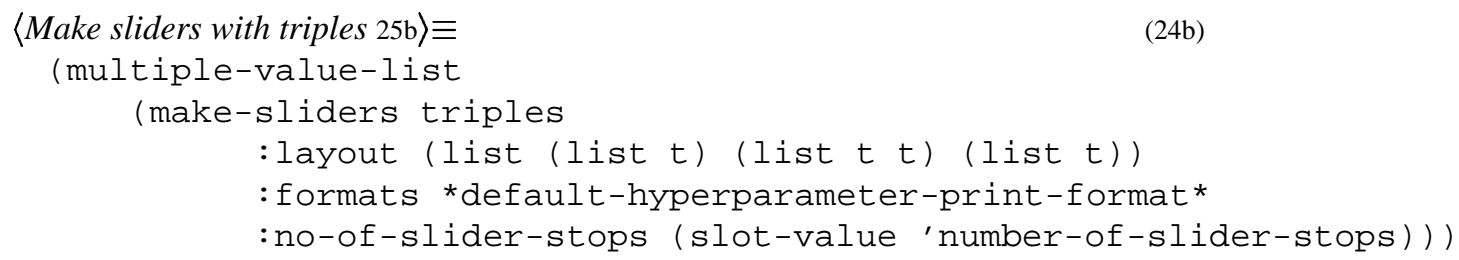

Uses *default-hyperparameter-print-format * 43a.

Finally, we create a decent title, become alert once again as opposed to being lazy and then synchronize everything and show those windows.

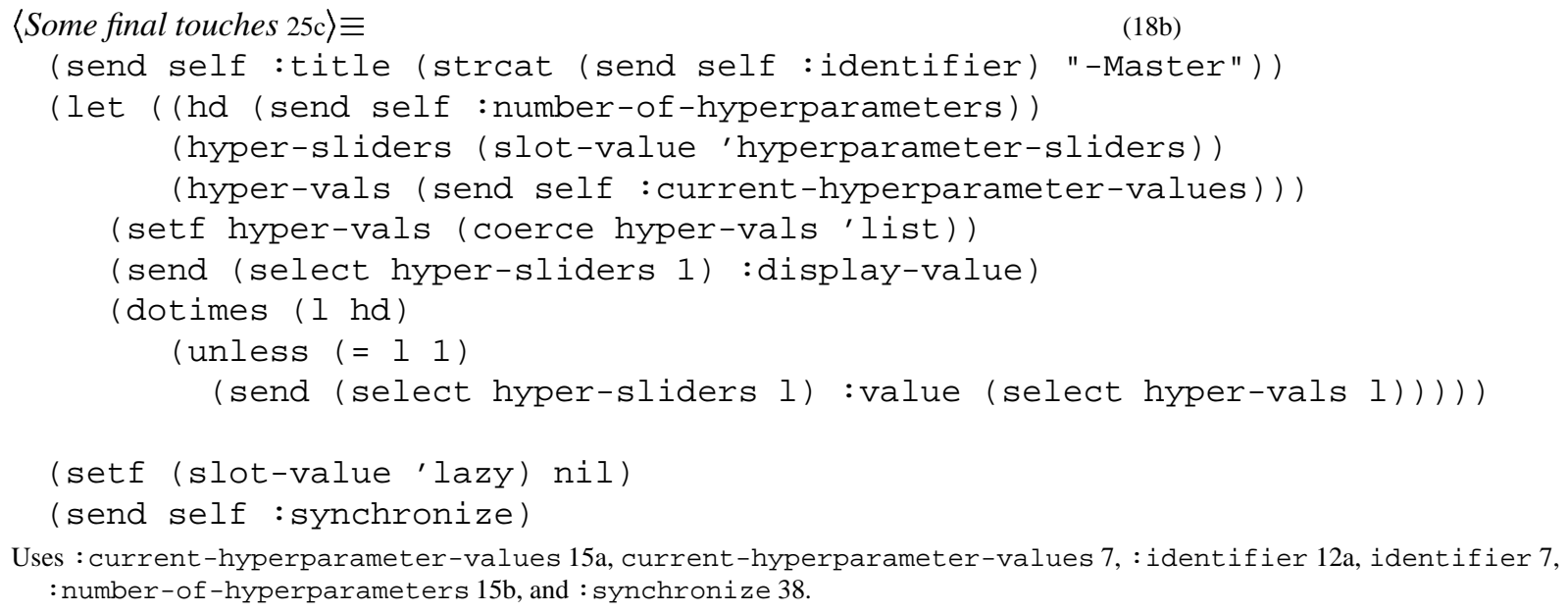


July 2, 1998

bsa.nw

\subsection{The : process-run-file Method}

In handling the "run" file, note the assumptions made about the file structure and how some irrelevant headings are expected and skipped.

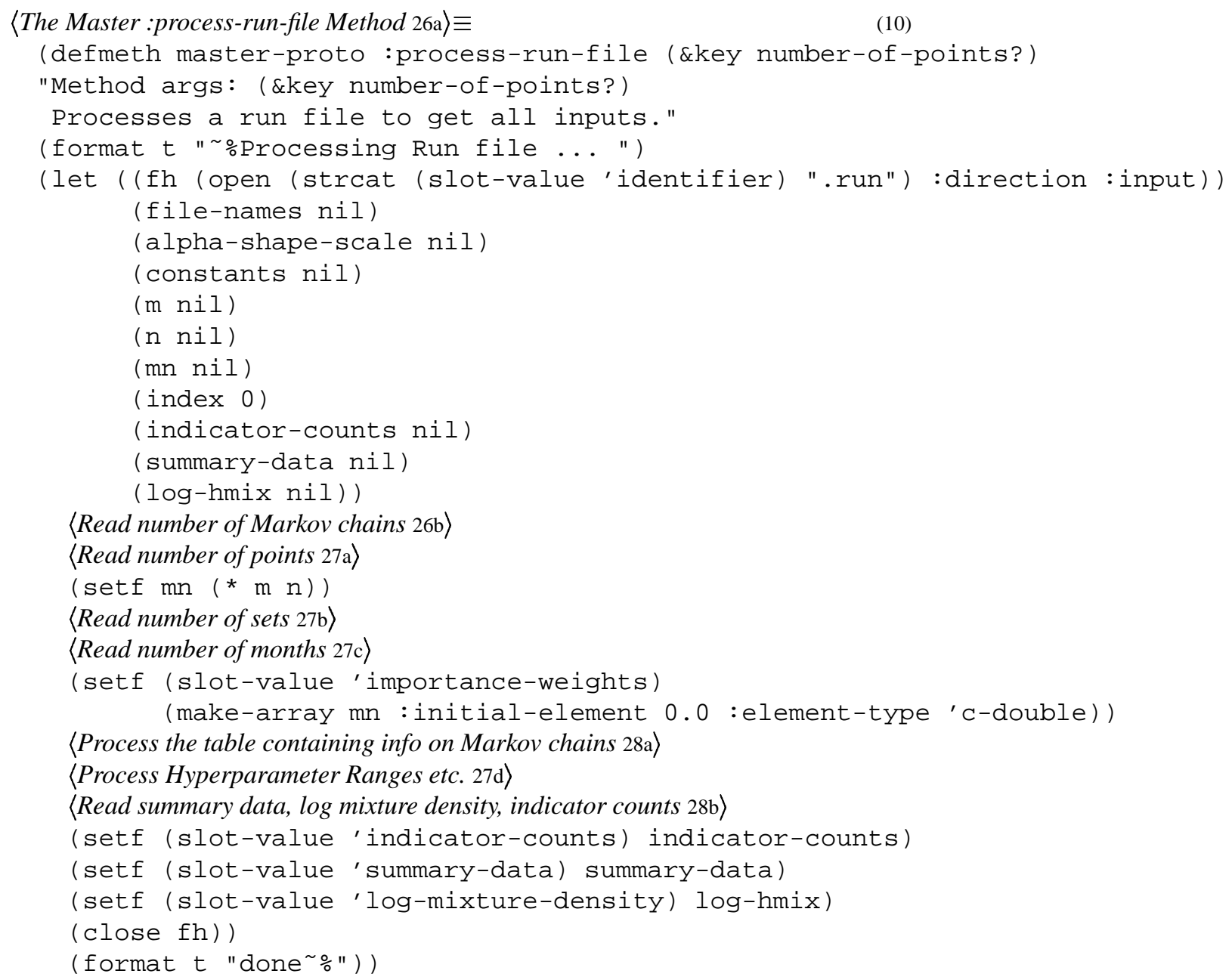

Uses indicator-counts 7, master-proto 7, number-of-points 7, :process-run-file 28b, and summary-data 7.

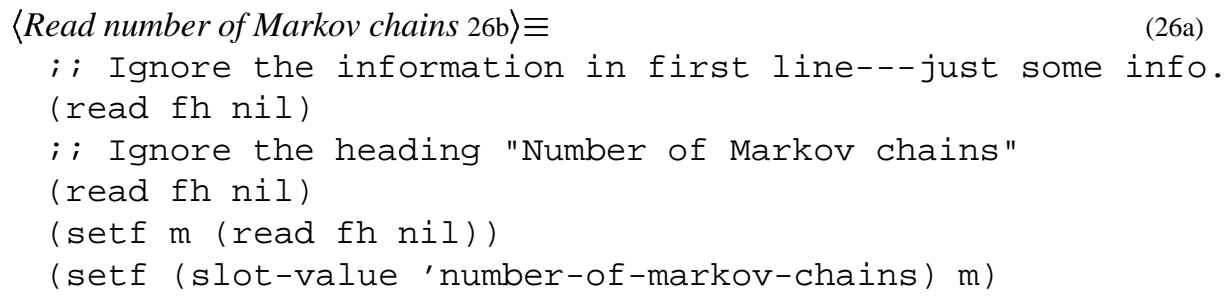


Uses number-of-points 7 .

27c $\langle$ Read number of months $27 \mathrm{c}\rangle \equiv$

; ; Ignore the heading "Number of Months"

(read fh nil)

(setf (slot-value 'number-of-months) (read fh nil))

27d $\langle$ Process Hyperparameter Ranges etc. $27 \mathrm{~d}\rangle \equiv$

(26a) ; ; Ignore the leading line (read fh nil)

(let ( (range nil)

(r (iseq 3))

(s (iseq 3))

(i (iseq 3)) )

(dotimes ( $j 3$ )

(read fh nil) (read fh nil)

(setf range (cons (read fh nil) nil))

(read fh nil)

(setf range (cons (read fh nil) range))

(setf (select $r j$ ) (reverse range))

(read fh nil)

(setf (select $i j)$ (read fh nil))

(read fh nil)

(setf (select $s j)$ (read fh nil)))

(send self :hyperparameter-ranges

(append $r$

(list (list 0 (1- (slot-value 'number-of-months $)))))$ )

(setf (slot-value 'number-of-slider-stops)

(append s (list (slot-value 'number-of-months))))

(setf (slot-value 'initially-specified-hyperparameter-values)

(append $i$ (list (* 0.5 (slot-value 'number-of-months))))))

Uses : hyperparameter-ranges $16 \mathrm{~b}$ and hyperparameter-ranges 7. 
; ; Now read the table of file name, alpha,

; $\quad$ shape, scale and log constant after ignoring the heading.

(read fh nil)

(dotimes ( $i \mathrm{~m}$ )

(setf file-names (cons (read fh nil) file-names))

(setf alpha-shape-scale (cons (read fh nil) alpha-shape-scale))

(setf alpha-shape-scale (cons (read fh nil) alpha-shape-scale))

(setf alpha-shape-scale (cons (read fh nil) alpha-shape-scale))

(setf constants (cons (read fh nil) constants)))

(setf (slot-value 'data-file-names) (reverse file-names))

(setf (slot-value' log-constants-of-proportionality)

(if $(=\mathrm{m} 1)$

(make-array 1 :initial-element 0

:element-type 'c-double)

(make-array $(1-\mathrm{m})$ : initial-contents (rest (reverse constants)) :element-type 'c-double)))

(setf (slot-value 'hyperparameters-used-in-markov-chains)

(make-array (list $\mathrm{m}$ 3)

:initial-contents (reverse alpha-shape-scale)

:element-type 'c-double))

Defines:

: process-run-file, used in chunks 19a and 26a.

Uses indicator-counts 7 and summary-data 7. 


\subsection{The :process-frequency-table Method}

$29 \mathrm{a}$

$\langle$ The Master :process-frequency-table Method 29a〉三 (defmeth master-proto :process-frequency-table (fh nd ind)

"Args: (fh nd ind)

This is a convenience method used in reading the data files. It reads

the frequency table of size nd from fh, the file stream, and fills

the indicator-counts slot at index ind."

(let ( $\mathrm{x}$-vals (make-array nd))

(freq (make-array nd))

(sort-index nil)

(indicator-counts (slot-value 'indicator-counts))

(number-of-months (slot-value 'number-of-months))

$(j \mathrm{j})$

$\left(\begin{array}{ll}\mathrm{k} & 0\end{array}\right)$

( freq-sum 0)

(smallest-month 0))

$\langle$ Read frequency table and sort values $29 \mathrm{~b}\rangle$

(dotimes (i nd)

( setf smallest-month

〈Find smallest month not less than $i$-th ordered $x$-val 29c〉)

(when smallest-month

〈Fill table entries with current frequency sum 29d〉

〈Bump starting index for next stretch 29e

(Update frequency sum 30a) ) )

〈Handle situation when $x$-values run out before month values $30 \mathrm{~b}\rangle)$ )

Defines:

: process-frequency-table, used in chunk 34c.

Uses indicator-counts 7, master-proto 7, and number-of-months 7.

$\langle$ Read frequency table and sort values $29 \mathrm{~b}\rangle \equiv$

(dotimes ( 1 nd)

(setf (aref $x$-vals l) (read fh nil))

(setf (aref freq l) (read fh nil)))

(setf sort-index (make-array nd : initial-contents (order x-vals)) )

In the code snippet below, nil is returned if no such month is found.

$\langle$ Find smallest month not less than $i$-th ordered $x$-val $29 \mathrm{c}\rangle \equiv$

( loop

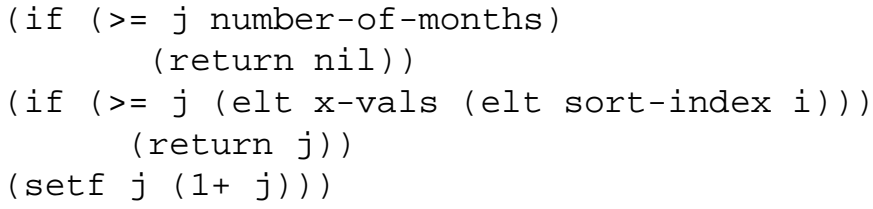

Uses number-of-months 7 .

$\langle$ Fill table entries with current frequency sum 29d $\rangle$

(dotimes ( $\mathrm{x}$ (- smallest-month $\mathrm{k}$ ))

(setf (aref indicator-counts ind (+ $\mathrm{x}$ k)) freq-sum))

Uses indicator-counts 7. 
July 2, 1998

bsa.nw

$30 \mathrm{a}$

$\langle$ Update frequency sum 30a $\rangle$

(setf freq-sum (+ freq-sum (elt freq (elt sort-index i))))

$30 \mathrm{~b}\langle$ Handle situation when $x$-values run out before month values $30 \mathrm{~b}\rangle \equiv$

(dotimes (x (- number-of-months k))

$$
\text { (setf (aref indicator-counts ind ( } x \text { k)) freq-sum)) }
$$

Uses indicator-counts 7 and number-of-months 7 . 
July 2, 1998

bsa.nw

\subsection{The : graphical-interface Method}

$31\langle$ The Master : graphical-interface Method 31〉三

(defmeth master-proto :graphical-interface

(\&key identifier?

number-of-markov-chains?

number-of-points?

file-names?

log-constants?)

"Method args: (\&key identifier?

number-of-chains?

number-of-points

file-names?

log-constants?)

Sets up a graphical interface for some inputs if not specified."

(let* ( (id (if identifier?

identifier?

(Get an identifier 32a) ) )

(m (if number-of-markov-chains?

number-of-markov-chains?

〈Get the number of Markov chains 32b $\rangle)$ )

(file-names (if file-names?

file-names?

$\langle$ Get data file names 33〉))

(n (if number-of-points?

number-of-points?

(Get the number of points 34a)) )

(initial-guess (if log-constants?

log-constants?

(if $(>\mathrm{m}$ 1)

(Get initial guess 34b )))) )

(setf (slot-value 'identifier) id)

(setf (slot-value 'data-file-names) file-names)

(setf (slot-value 'number-of-markov-chains) m)

(setf (slot-value 'number-of-points) n)

(setf (slot-value 'number-of-months) *default-number-of-months*)

(setf (slot-value 'indicator-counts)

(make-array (list (* $\mathrm{m} \mathrm{n}$ ) (slot-value 'number-of-months))

:element-type ' $\mathrm{C}$-long))

(setf (slot-value 'importance-weights)

(make-array (* $\mathrm{m} \mathrm{n}$ ) : initial-element 0 :element-type 'c-double))

(setf (slot-value' log-mixture-density)

(make-array (* $\mathrm{m} \mathrm{n})$ : initial-element 0

: element-type 'c-double))

(setf (slot-value 'log-constants-of-proportionality)

(if $(=\mathrm{m} 1)$

(make-array 1

:initial-element 0

:element-type 'c-double)

(make-array $(1-\mathrm{m})$

:initial-contents initial-guess 
July 2, 1998

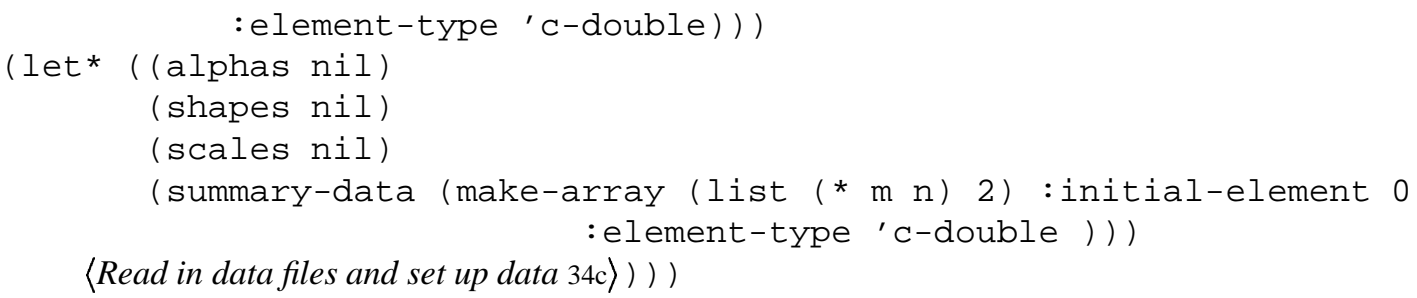

Uses *default-number-of-months * 43a, identifier 7, master-proto 7, number-of-markov-chains 7 , number-of-months 7, number-of-points 7 , and summary-data 7.

$\langle$ Get an identifier 32a $\rangle \equiv$

(get-nonempty-string-dialog

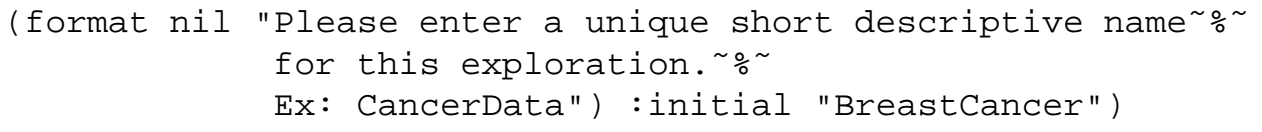

$\langle$ Get the number of Markov chains 32b $\rangle \equiv$

(select (get-tested-value-dialog

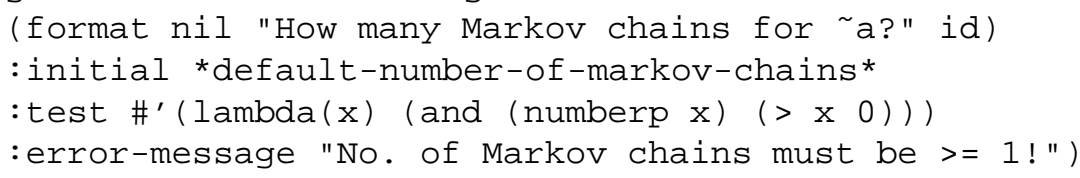

Uses *default-number-of-markov-chains* 43a and number-of-markov-chains 7. 
July 2, 1998

Uses *default-number-of-points * 43a and number-of-points 7.

To process the data, we use both the input and output file used in running the Markov chains.

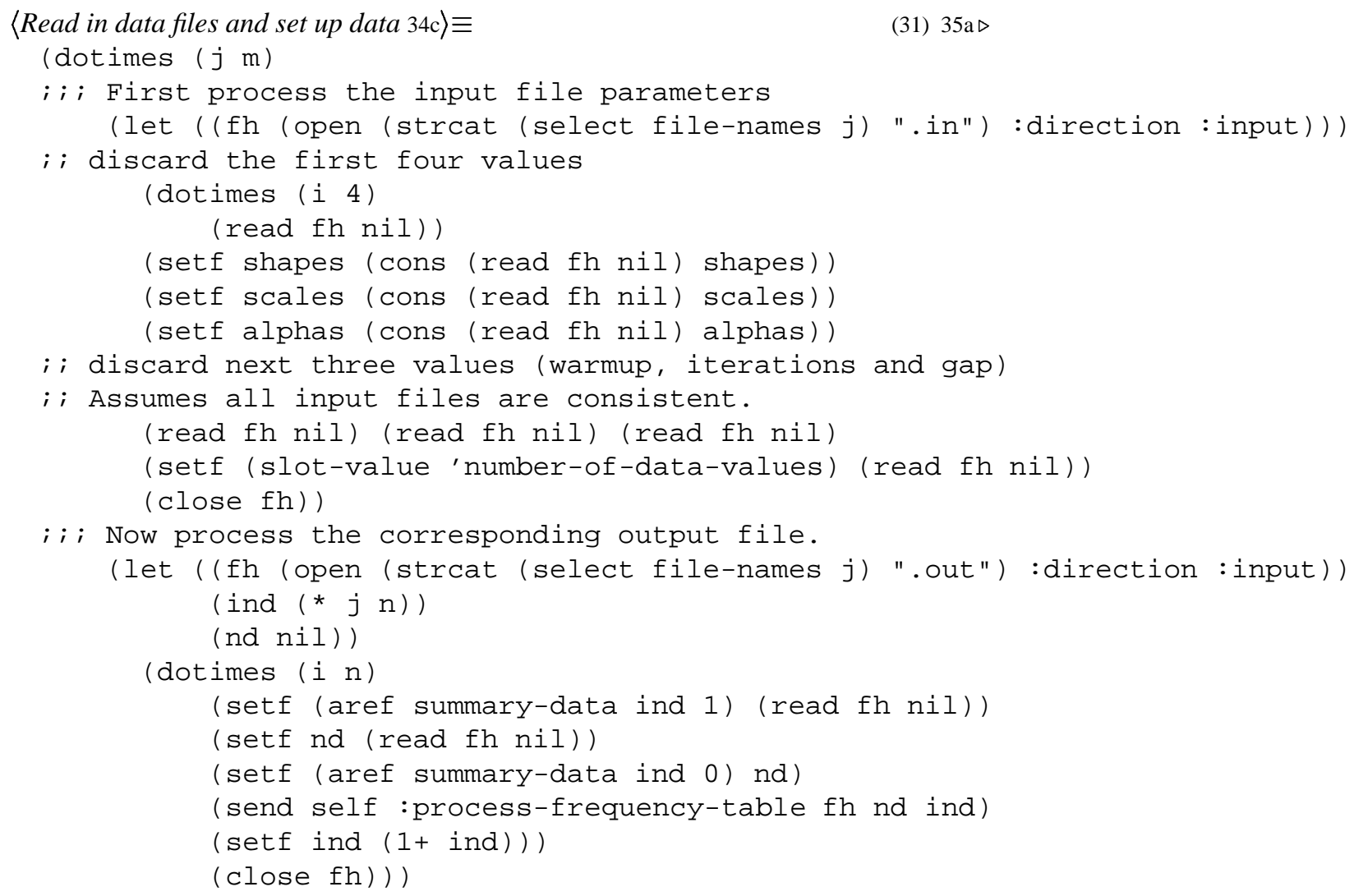

Uses : process-frequency-table 29a and summary-data 7. 
July 2, 1998

$\mathrm{bsa} \cdot \mathrm{nw}$

Now that all the Markov chain files have been processed, the slot-values can be set up.

$35 \mathrm{a}$

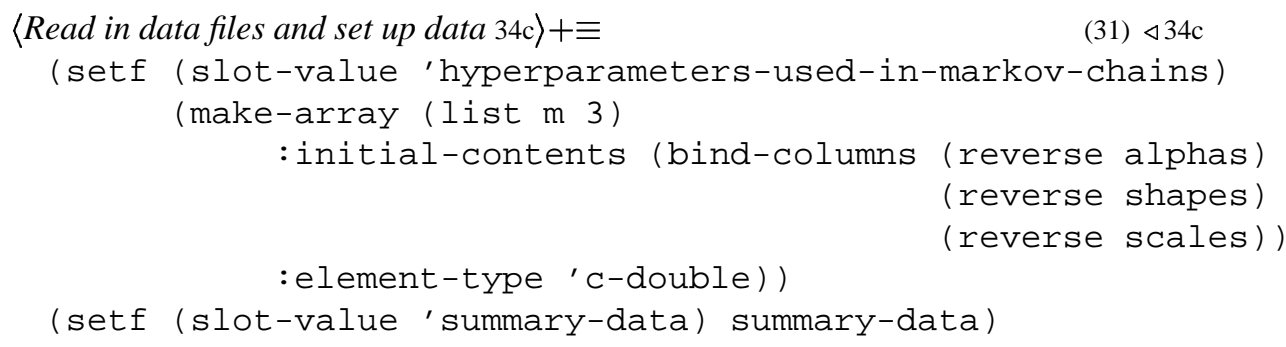

\subsection{The : create-run-file Method}

$\langle$ The Master :create-run-file Method 35b) $\equiv$

Uses indicator-counts 7, master-proto 7, and summary-data 7. 
Uses indicator-counts 7 and summary-data 7.

37c $\langle$ Show informative message 37c $\rangle \equiv$

(let ((id (slot-value' 'identifier))) 


\subsection{The : synchronize Method}

The : synchronize method is responsible for synchronizing the slaves so that the density estimates they display is for the current values of the hyperparameters. Thus, if any hyperparameter value is changed, the :synchronize method should be invoked.

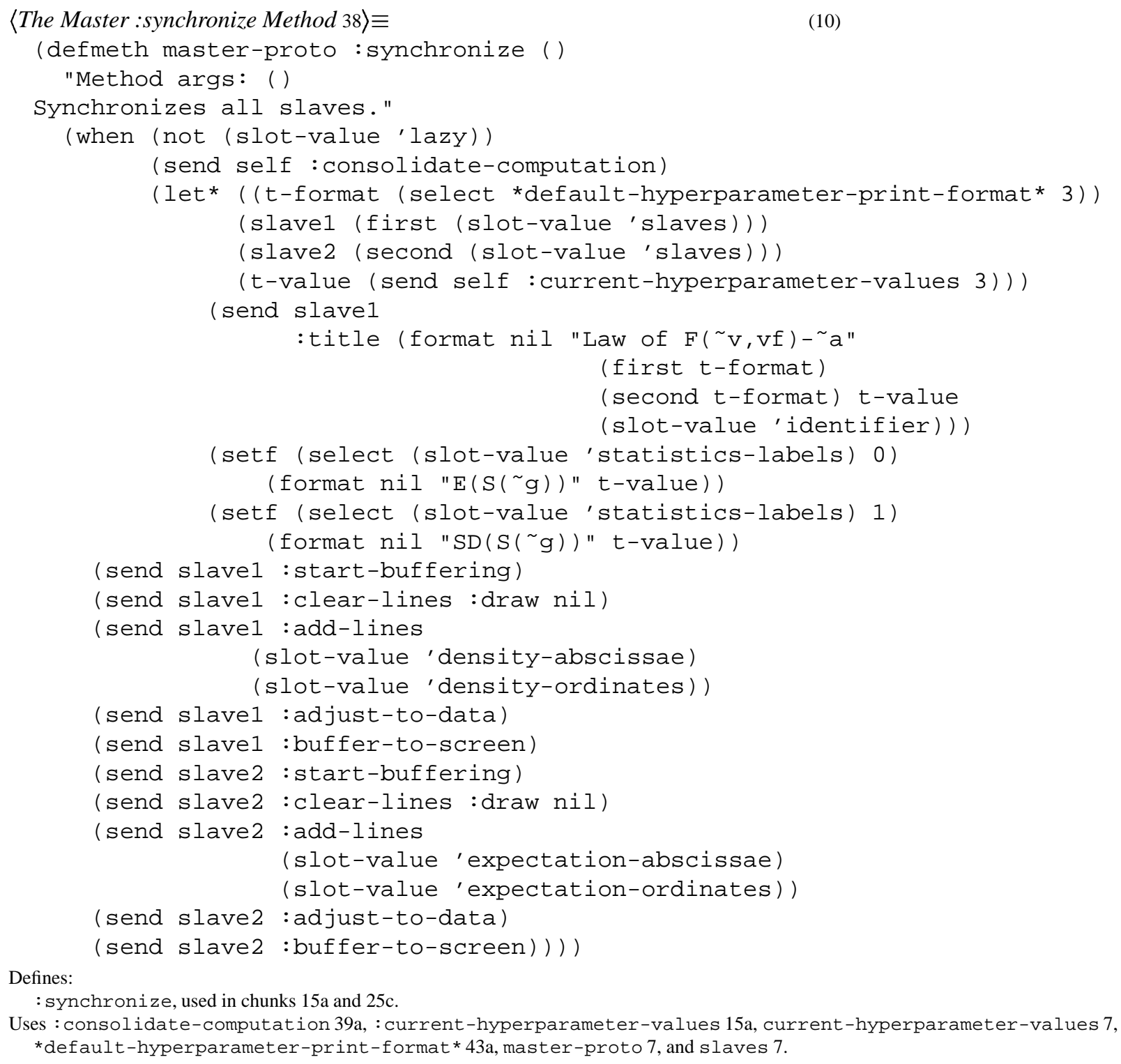




\subsection{The : consolidate-computation Method}

In this method we compute all the relevant statistics such as $E(S(t))$ and $\sigma_{S(t)}$ for various values of $t$, as well as the effective sample size. This method was added to consolidate all computations for acceptable performance and makes several other methods above superfluous.

$\langle$ The Master : consolidate-computation Method 39a) $\equiv$ (defmeth master-proto : consolidate-computation ()

"Method args: ()

Consolidates all computations for sake of speed. This method

recalculates the importance weights, computes relevant statistics and saves them in the slots."

(call-by-reference-oldcfun "consolidateComputation"

(slot-value 'shared-library) *default-number-of-plot-stops *

(slot-value 'statistics)

(slot-value 'density-abscissae) (slot-value 'density-ordinates)

(slot-value 'expectation-abscissae)

(slot-value 'expectation-ordinates)

(slot-value 'standard-deviation-ordinates)))

Defines:

: consolidate-computation, used in chunk 38.

Uses consolidateComputation 60, master-proto 7 , and statistics 750 .

\subsection{The : reset Method}

"Method args: ()

Resets the state of all objects."

(send self :current-hyperparameter-values

(send self :initially-specified-hyperparameter-values)))

Defines:

: reset, used in chunk $24 \mathrm{~b}$.

Uses : current-hyperparameter-values 15a, current-hyperparameter-values 7, : initially-specified-hyperparameter-value initially-specified-hyperparameter-values 7, and master-proto 7.

\subsection{The :effective-sample-size Method}

The actual computation of the effective sample size is now done in the $\mathrm{C}$ code that computes the importance weights (see section 6.9). We note that that the formula for computing effective sample size was originally used in estimating standard deviations in stratified sampling. It can be used to give ballpark figures, but should not be taken too literally.

Defines:

: effective-sample-size, never used.

Uses master-proto 7. 
July 2, 1998

\subsection{The :print-all-statistics Method}

(let* ((hyperparameter-names (slot-value 'hyperparameter-names))

(hyper-strings (send self :labelled-hyperparameter-values))

(max-name-len (max (mapcar \#' length hyperparameter-names))))

(format $t$ " \% \%

(format $t$ "Hyperparameter Settings: ${ }^{\circ} "$ )

(dolist (x hyper-strings)

(format $t$ " $\left.a \sim \frac{\circ}{0} " x\right)$ )

(format $t$ "Total Sample Size $=\sim 5 \mathrm{~d}$. $\% "$

(* (slot-value 'number-of-markov-chains)

(slot-value 'number-of-points)))

(format $t$ "Effective Sample Size = 5d. \%"

(select (slot-value 'statistics) 2)))

(dolist (x (slot-value 'slaves))

(send $x$ :print-summary))

(format $t$ " \%*** End of Statistics $\star \star \star \sim \% \sim \% ")$ )

Defines:

:print-all-statistics, used in chunks $24 \mathrm{~b}$ and 61.

Uses hyperparameter-names 7, : identifier 12a, identifier 7, : labelled-hyperparameter-values 40b, master-proto 7 , : print-summary 46a, and statistics 750.

\subsection{The : labelled-hyperparameter-values Method}

$\langle$ The Master :labelled-hyperparameter-values Method 40b〉三

(defmeth master-proto :labelled-hyperparameter-values ()

(let* ((hyper-names (slot-value 'hyperparameter-names))

(max-name-len (max (mapcar \#'length hyper-names)))) (mapcar

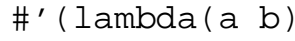

(let ( (y (select *default-hyperparameter-print-format* a)))

(if (listp y)

(format nil " va $=\sim \mathrm{v}, \mathrm{vf} "$ max-name-len

b (first $y)$ (second $y$ )

(send self :current-hyperparameter-values a))

(format nil (strcat " va = " y) max-name-len b

(send self :current-hyperparameter-values a)))))

(iseq (send self :number-of-hyperparameters)) hyper-names)))

Defines:

: labelled-hyperparameter-values, used in chunk 40a.

Uses : current-hyperparameter-values 15a, current-hyperparameter-values 7, *default-hyperparameter-print-format*43a, master-proto 7, and : number-of-hyperparameters $15 \mathrm{~b}$. 
July 2, 1998

bsa.nw

\subsection{The : statistics Method}

The : statistics method for the master returns the value of the slot statistics.

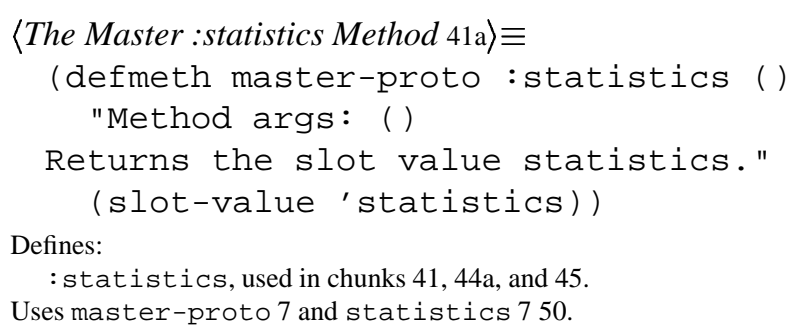

\subsection{The :statistics-print-formats Method}

The :statistics-print-formats method for the master returns the value of the slot statistics-print-formats.

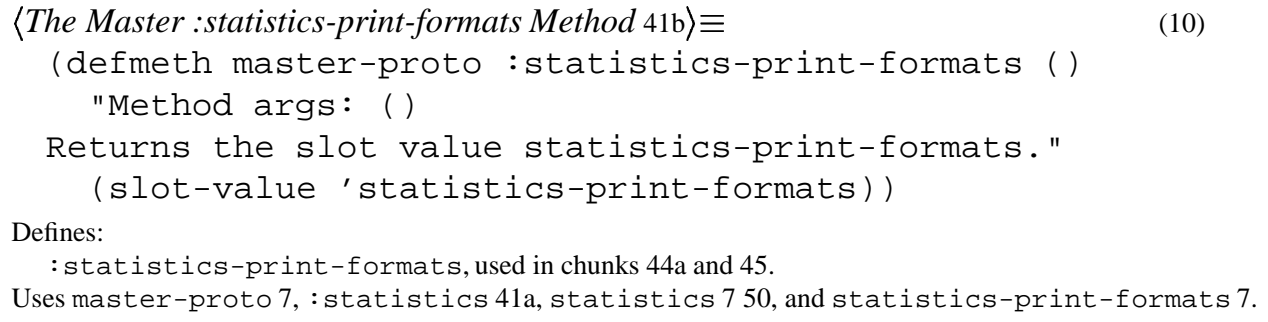

\subsection{The : statistics-labels Method}

The :statistics-labels method for the master returns the value of the slot statistics-labels.

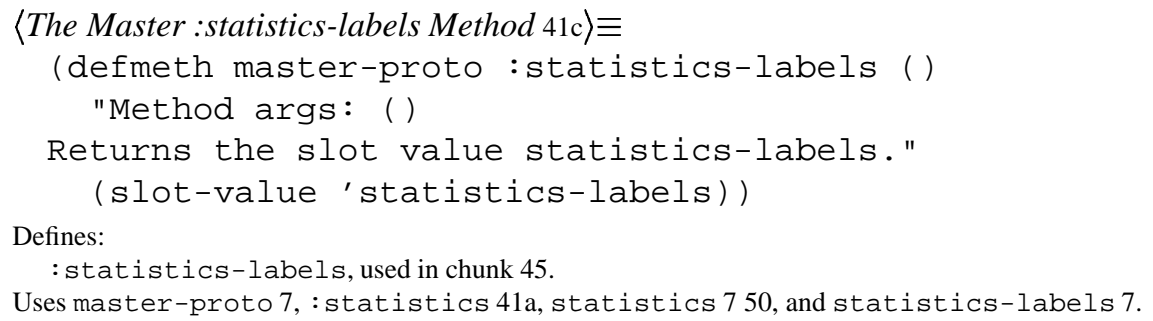


July 2, 1998

bsa.nw

\subsection{The : toggle-timing Method}

This method toggles timing on and off.

$42 \mathrm{a}$

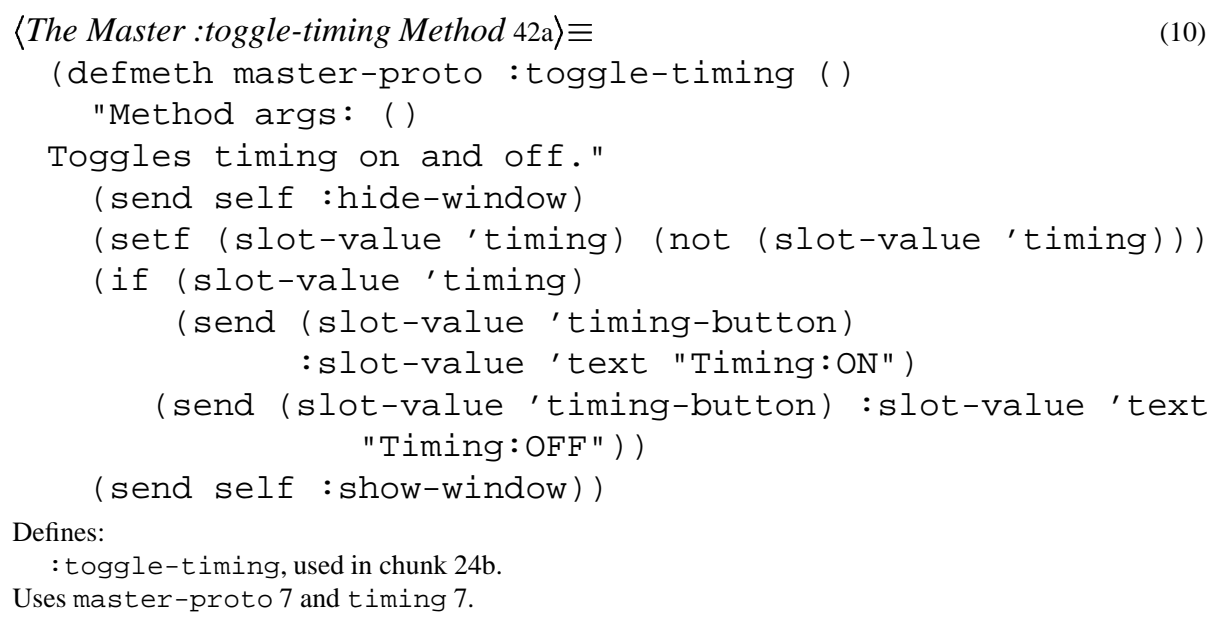

\subsection{The : superimpose Method}

This method returns the value of the superimpose slot.

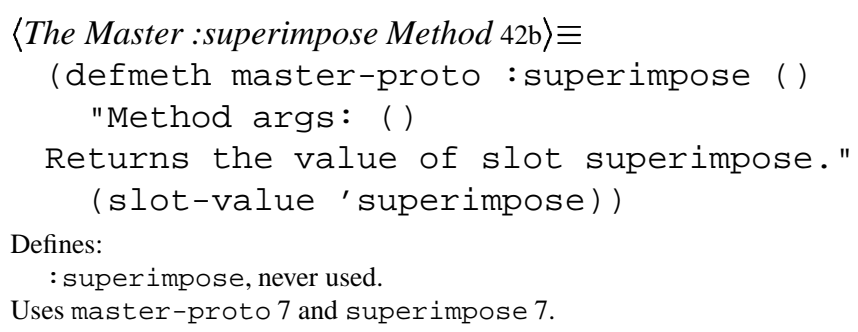

\subsection{The : close Method}

The : close method for the master must close the slaves window that are active. Finally, it must commit hara-kiri.

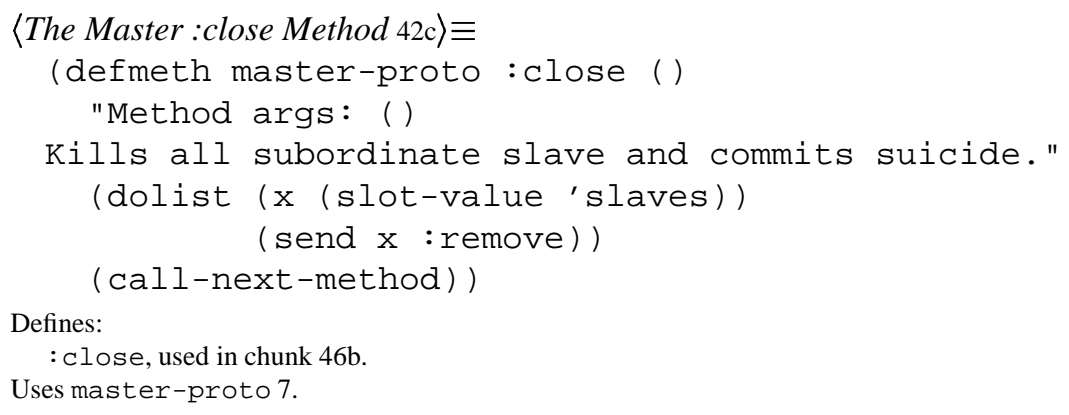




\subsection{Defaults for Master}

We shall use the prefix "BSA" for Bayesian Sensitivity Analysis.

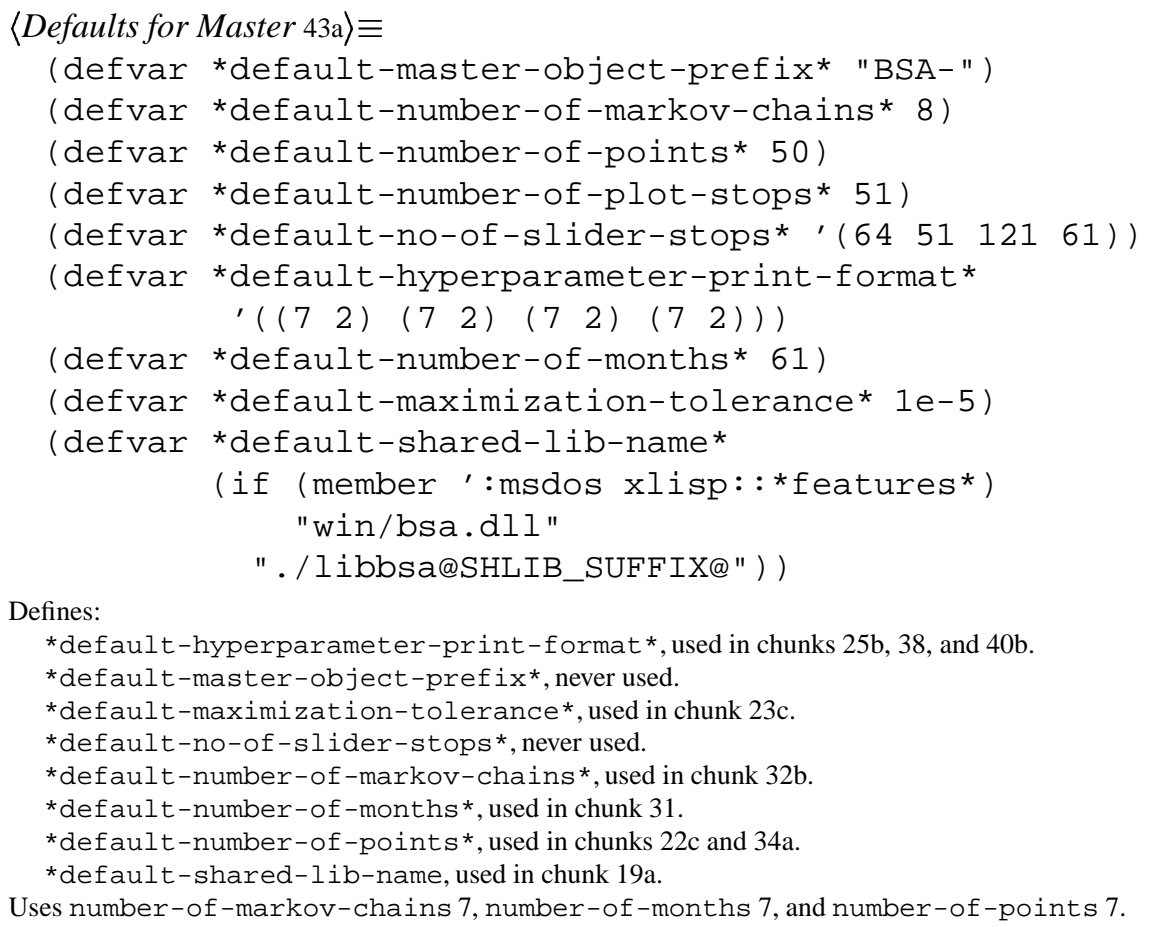

\section{The Slave Object Prototype}

The Slave prototype inherits from scatterplot-proto.

(defproto slave-proto ' (master survival-plot color-index)

() scatterplot-proto

"The Slave prototype. Master is its master upon whom the slave relies for all data. Survival-plot is nil or t indicating what is to be plotted. Color-index is used in superimposition.")

$\langle$ Methods for Slave Prototype 43c

$\langle$ Defaults for Slave 47a〉

Defines:

slave-proto, used in chunks $24 \mathrm{a}$ and 44-46.

The methods for slave-proto follow.

〈The Slave :isnew Method 44a)

$\langle$ The Slave :redraw-background Method 44b

〈The Slave :redraw-statistics Method 45〉

〈The Slave :print-summary Method 46a〉

$\langle$ The Slave :close Method 46b 
July 2, 1998

$\mathrm{bsa} \cdot \mathrm{nw}$

44

\subsection{The : isnew Method}

$44 \mathrm{a}$

$\langle$ The Slave :isnew Method 44a $\equiv$

$(43 c)$

(defmeth slave-proto :isnew (master \&key (go-away t) (survival-plot nil))

"Method args: master \&rest args

Creates a new instance of the slave-proto object."

(setf (slot-value 'master) master)

(setf (slot-value 'survival-plot) survival-plot)

(setf (slot-value 'color-index) 0)

(call-next-method 2 :go-away go-away :draw nil)

(if (not (slot-value 'survival-plot))

(let ((skip (+ (send self :text-ascent) (send self :text-descent)))

(len (length (send master :statistics-print-formats))))

(send self :margin

0 (+ (* len skip) (send self :text-descent)) 00

: draw nil)))

(send self : redraw))

Defines:

: isnew, used in chunk 51.

Uses slave-proto 43b, : statistics 41a, statistics 750 , : statistics-print-formats 41b, and statistics-print-formats 7.

\subsection{The : redraw-background Method}

$\langle$ The Slave :redraw-background Method $44 \mathrm{~b}\rangle \equiv$

(defmeth slave-proto :redraw-background ()

"Method args: ()

Redraws the background of the screen"

(send self : start-buffering)

(call-next-method)

(unless (slot-value 'survival-plot)

(send self :redraw-statistics))

(send self :buffer-to-screen))

Defines:

: redraw-background, never used.

Uses : redraw-statistics 45, slave-proto 43b, and statistics 750. 
July 2, 1998

bsa.nw

\subsection{The : redraw-statistics Method}

(defmeth slave-proto :redraw-statistics ()

"Method args: ()

Redraws the statistics on the screen"

(let* ((master (slot-value 'master))

(ascent (send self :text-ascent))

(descent (send self :text-descent))

(skip (+ ascent descent))

(em (send self :text-width "m"))

(en (send self :text-width "n"))

(canvas-width (send self :canvas-width))

(y 0 )

(stats-labels (send master :statistics-labels))

(name-width (max (mapcar \#' (lambda $(x)$ (send self :text-width $x$ )) stats-labels)) )

(value-strings (mapcar \#' (lambda (x y)

(format nil $\mathrm{x} y$ ))

(send master :statistics-print-formats)

(coerce (send master :statistics) 'list)))

(value-width (max (mapcar \#' (lambda (x)

(send self :text-width $x$ ))

value-strings)) )

(x2 (- canvas-width value-width em))

(x1 (- x2 name-width em)))

(dotimes (i (length stats-labels))

(let ( $(s)$ (select stats-labels $i))$

(sv (select value-strings i)))

$(\operatorname{set} \mathrm{y}(+\mathrm{y}$ skip) $)$

(send self :draw-text sl x1 y 0 0)

(send self : draw-text sv x2 y 0 0)))) )

Defines:

:redraw-statistics, used in chunk $44 \mathrm{~b}$

Uses slave-proto 43b, :statistics 41a, statistics 750 , :statistics-labels 41c, statistics-labels 7 , :statistics-print-formats $41 \mathrm{~b}$, and statistics-print-formats 7. 
July 2, 1998

bsa.nw

\subsection{The : print-summary Method}

This method basically calculates the mean and variance of beta distributions.

Defines:

: print-summary, used in chunk 40a.

Uses : current-hyperparameter-values 15a, current-hyperparameter-values 7, and slave-proto 43b.

\subsection{The : close Method}




\subsection{Defaults for Slave}

(defvar *default-slave-plot-stops * 51)

(defvar *default-statistics-labels *

' ("E(S(t))" "SD(S(t))" "Eff. Sample Size"))

(defvar *default-statistics-print-formats *

' (" $5,3 f "$ " $5,3 f "$ " $5,0 f ")$ )

Defines:

*default-slave-plot-size*, never used.

*default-slave-plot-stops*, never used.

*default-statistics-labels*, used in chunk 19a.

*default-statistics-print-formats ${ }^{*}$, used in chunk 19a.

Uses statistics 7 50, statistics-labels 7 , and statistics-print-formats 7.

\section{The C Programs}

In designing the $\mathrm{C}$ programs, we assume one thing-that no compaction is done during the lifetime of the master object. This allows us to refrain from passing pointers to data arrays each time a routine is called. Instead, all required pointers could be passed once when the master object is created and they remain fixed for the life of the master. Current versions of Lisp-Stat don't do memory compaction or move objects around.

Here's our copyright for C programs.

Copyright (C) 1994, 1995, 1998. Doss and Narasimhan

$\star * *$ Hani J. Doss (dossestat.ohio-state.edu) and

$\star \star \star$ B. Narasimhan (narasestat.stanford.edu)

$\star \star \star$

*** This program is free software; you can redistribute it and/or modify

$\star * *$ it under the terms of the GNU General Public License as published by

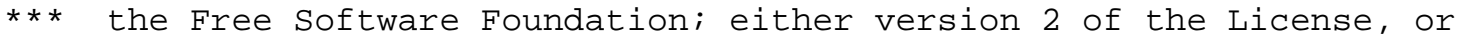

$\star \star *$ (at your option) any later version.

$\star \star \star$

$\star \star *$ This program is distributed in the hope that it will be useful,

$\star \star *$ but WITHOUT ANY WARRANTY; without even the implied warranty of

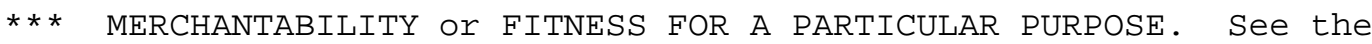

$\star \star \star$ GNU General Public License for more details.

$\star \star \star$

$\star \star \star$ You should have received a copy of the GNU General Public License

** along with this program; if not, write to the Free Software

$\star \star \star$ Foundation, Inc., 675 Mass Ave, Cambridge, MA 02139, USA.

$\star \star /$ 
Our $\mathrm{C}$ routines follow.

$48 \mathrm{a}$

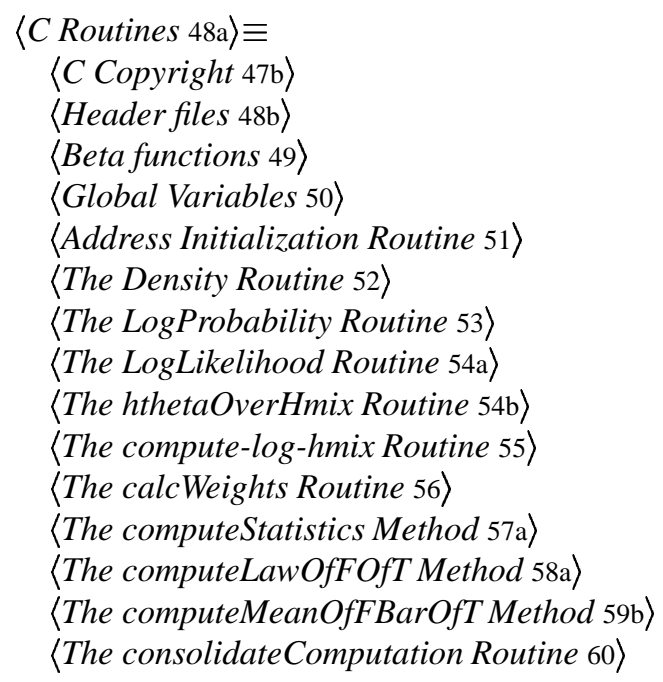

Here are the headers files we will need. Note the declaration for the prototype of the gamma function that comes with Lisp-Stat. This function is not declared static in the Lisp-Stat source and is therefore available to us. 


\subsection{Beta Functions}

We need two other functions, betdens and logbeta are indeed declared static in the Lisp-Stat source. Therefore, a copy of these routines is needed. In Lisp-Stat, the $\ln \Gamma$ function is known as mygamma.

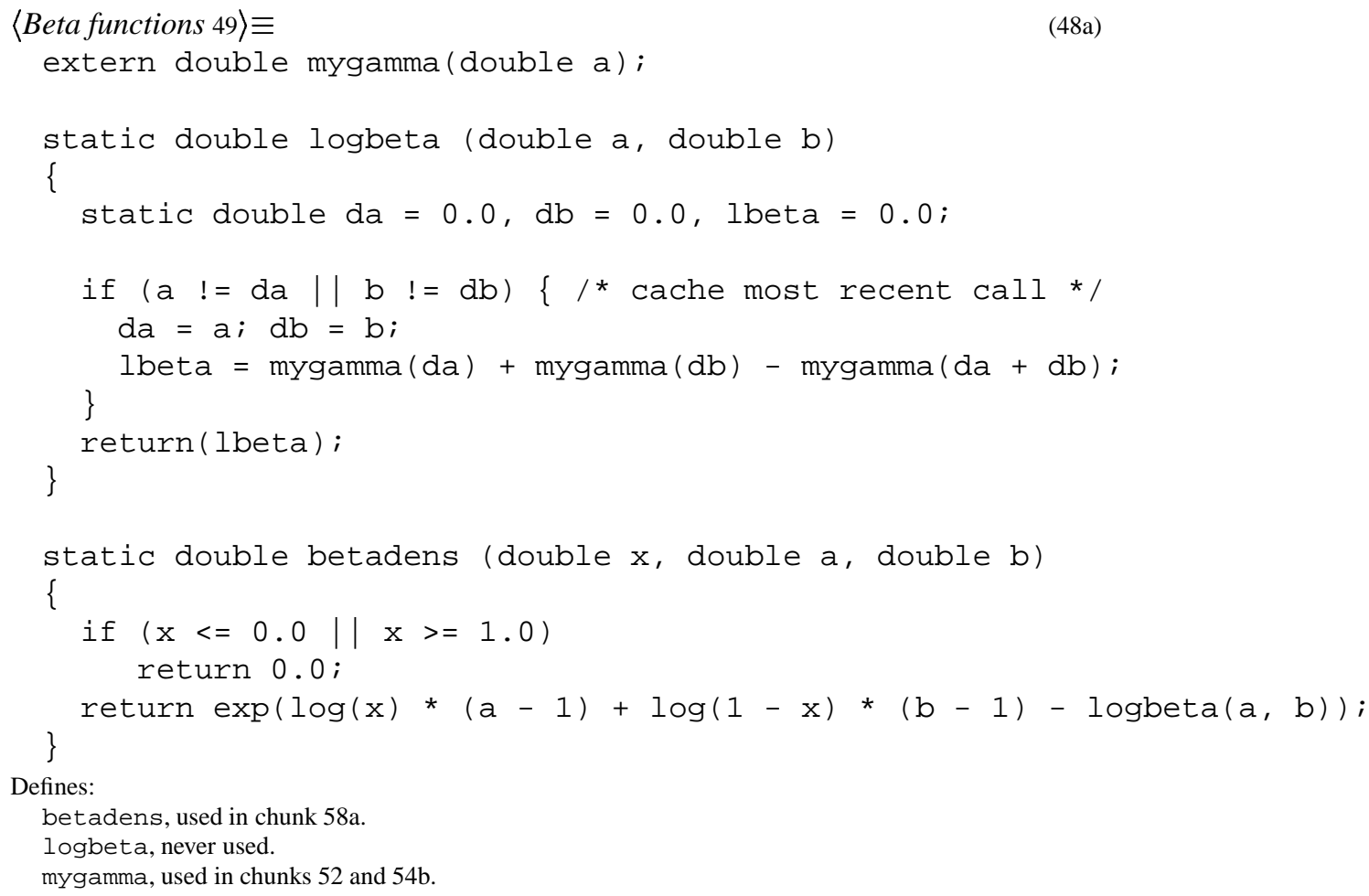




\subsection{Global Variables}

We begin with some global variables for all these $\mathrm{C}$ routines. Note that these are declared static. 


\subsection{Initialization Routine}

This initialization routine gathers addresses and stores them a static storage area. On the Windows platform, we also need to export the routines defined here.

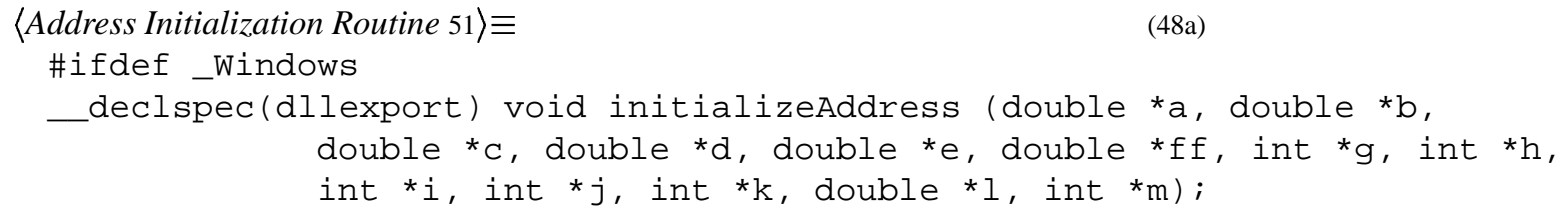

\#endif

$/ \star$

* Initialization routine, must be called as soon as the slots

* are created in the master : isnew method.

$\star$

void initializeAddress (argSummaryData, argHyperValuesUsedinMarkovChains, argCurrentHyperValues, argImportanceWeights, argLogMixtureDensity, argLogConstantsofProportionality, argNumberOfMarkovChains, argNumberOfPoints, argNumberofMonths, argNumberOfDataValues, argIndicatorCounts, argStatistics, argNumberofPointsForConstants) double *argSummaryData, *argHyperValuesUsedinMarkovChains, *argCurrentHyperValues, *argImportanceWeights, *argLogMixtureDensity, *argLogConstantsOfProportionality; double *argstatistics;

int *argNumberOfMarkovChains, *argNumberofPoints, *argNumberOfMonths, *argNumberOfDataValues, *argIndicatorCounts, *argNumberOfPointsForConstants;

\{ summaryData = argSummaryData; hyperValuesUsedInMarkovChains = argHyperValuesUsedinMarkovChains; currentHyperValues = argCurrentHyperValues; importanceWeights = argImportanceWeights; logMixtureDensity = argLogMixtureDensity; logConstantsOfProportionality = argLogConstantsOfProportionality; indicatorCounts = argIndicatorCounts;

numberOfMarkovChains $=$ *argNumberOfMarkovChains;

numberofPoints $=$ *argNumberofPoints;

numberOfMonths $=$ *argNumberOfMonths;

numberOfDataValues $=$ *argNumberOfDataValues;

statistics = argstatistics;

numberOfPointsForConstants $=$ *argNumberOfPointsForConstants;

\}

Defines:

initializeAddress, used in chunk 23a.

Uses current HyperValues 50, importanceWeights 50, indicatorCounts 50, : isnew 18b 44a, logConstant sOfProportionality 50, logMixtureDensity 50, numberOfDataValues 50, numberOfMarkovChains 50, numberOfMonths 50, numberOfPoints 50, numberOfPointsForConstants 50, statistics 7 50, and summaryData 50. 


\subsection{The C Equivalent of : $f$ Method}

This routine calculates the $j$-th posterior density at a point. See $:$ f method for master-proto. The formulas used for this and the next three methods all follow the development in [3].

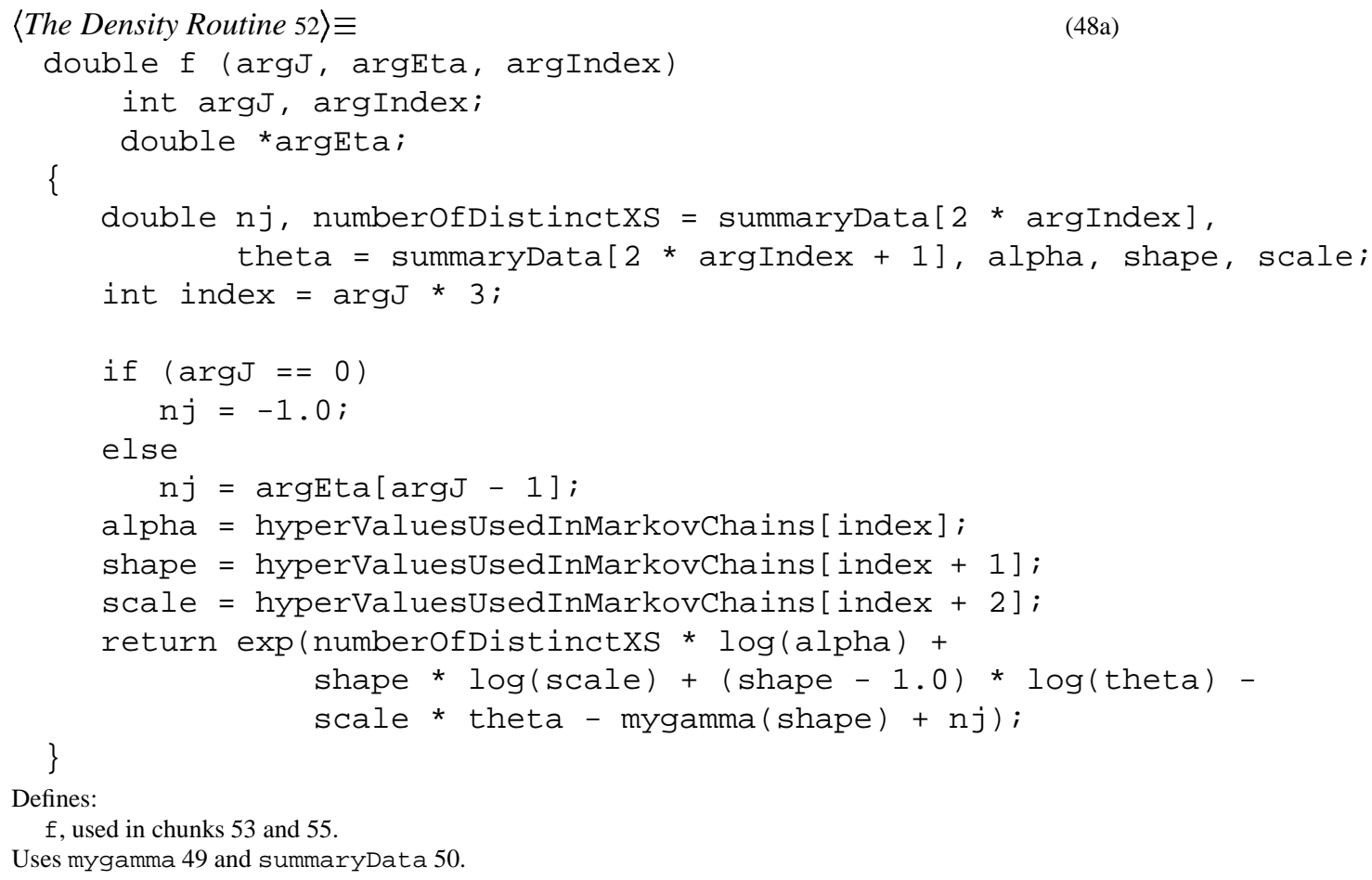


July 2, 1998

bsa.nw

\subsection{The C Equivalent of : logp Method}

This routine calculates the probability that a given point comes from a particular Markov chain. See : logp method for master-proto.

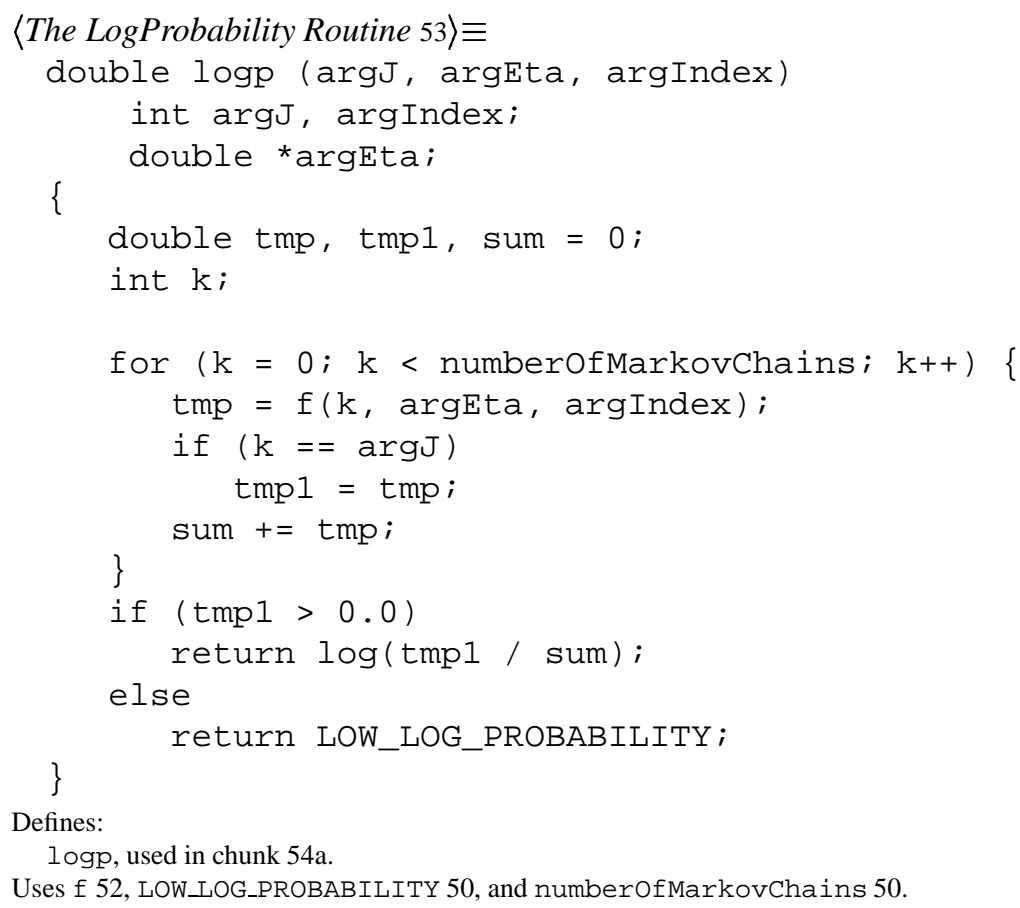


July 2, 1998

bsa.nw

\subsection{The C Equivalent of : loglik Method}

The routine that calculates the $\log$-likelihood. See $: \log l i k$ method for master-proto.

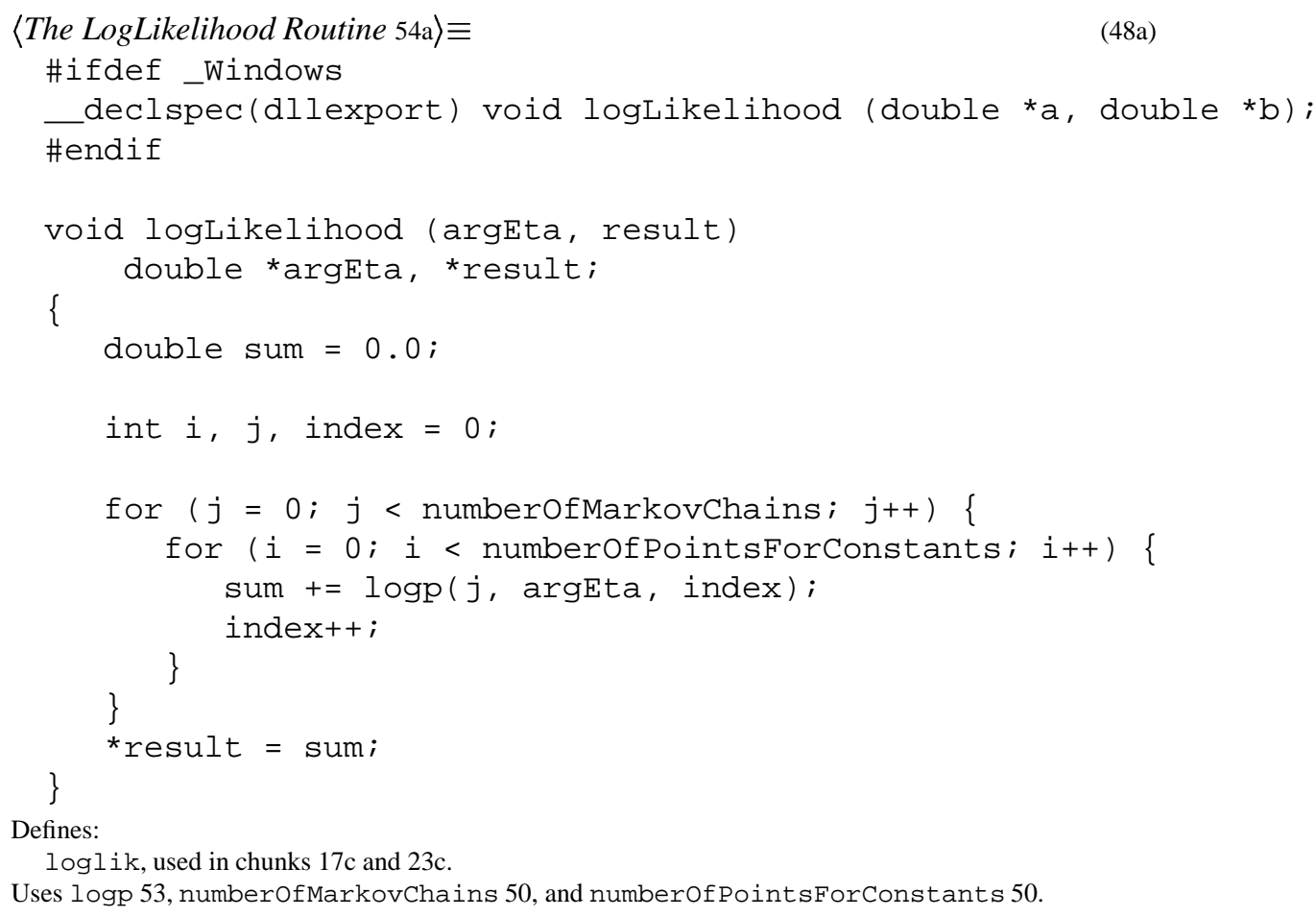

\subsection{The C Equivalent of : htheta-over-hmix Method}

See : htheta-over-hmix method for master-proto.

54b 〈The hthetaOverHmix Routine 54b〉三 double hthetaOverHmix (argIndex) 
July 2, 1998

bsa.nw

\subsection{The C Equivalent of : compute-log-hmix Method}

See : compute-log-hmix method for master-proto.

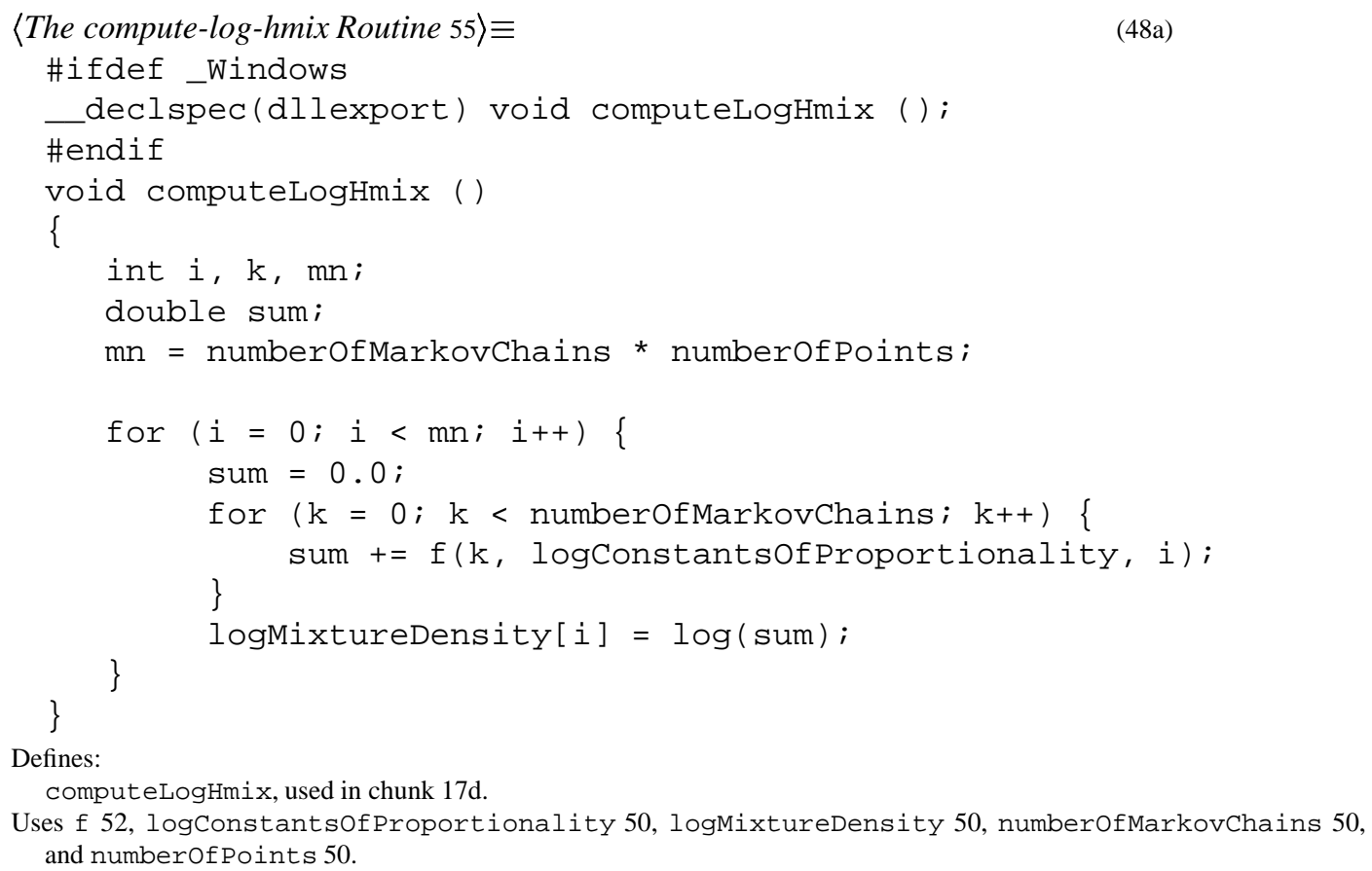


July 2, 1998

bsa.nw

\subsection{The C Equivalent of : calc-weights Method}

See :calc-weights method for master-proto.

Uses hthetaOverHmix 54b, importanceWeights 50, numberofMarkovChains 50, numberOfPoints 50 , and statistics 750. 
July 2, 1998

\subsection{The C Equivalent of : compute-law-of-f-of-t Method}

See : compute-law-of-f-of-t method for slave-proto. Note the difference in that some parameters need to be passed in this $C$ routine unlike the Lisp routine.

This method basically calculates for equally spaced values of $u$ in $[0,1]$ the weighted sum

$$
\sum_{g=1}^{m n} w_{g}^{(\kappa)} \beta\left(\alpha_{\theta(g)}^{(\kappa)}(t)+\sum_{i=1}^{n} \delta_{X_{i}^{(g)}}(t), M_{\theta^{(g)}}^{(\kappa)}+n-\alpha_{\theta^{(g)}}^{(\kappa)}(t)-\sum_{i=1}^{n} \delta_{X_{i}^{(g)}}(t)\right)(u),
$$

where $\beta(a, b)(u)$ denotes the beta density with parameters $a$ and $b$ evaluated at $u$.

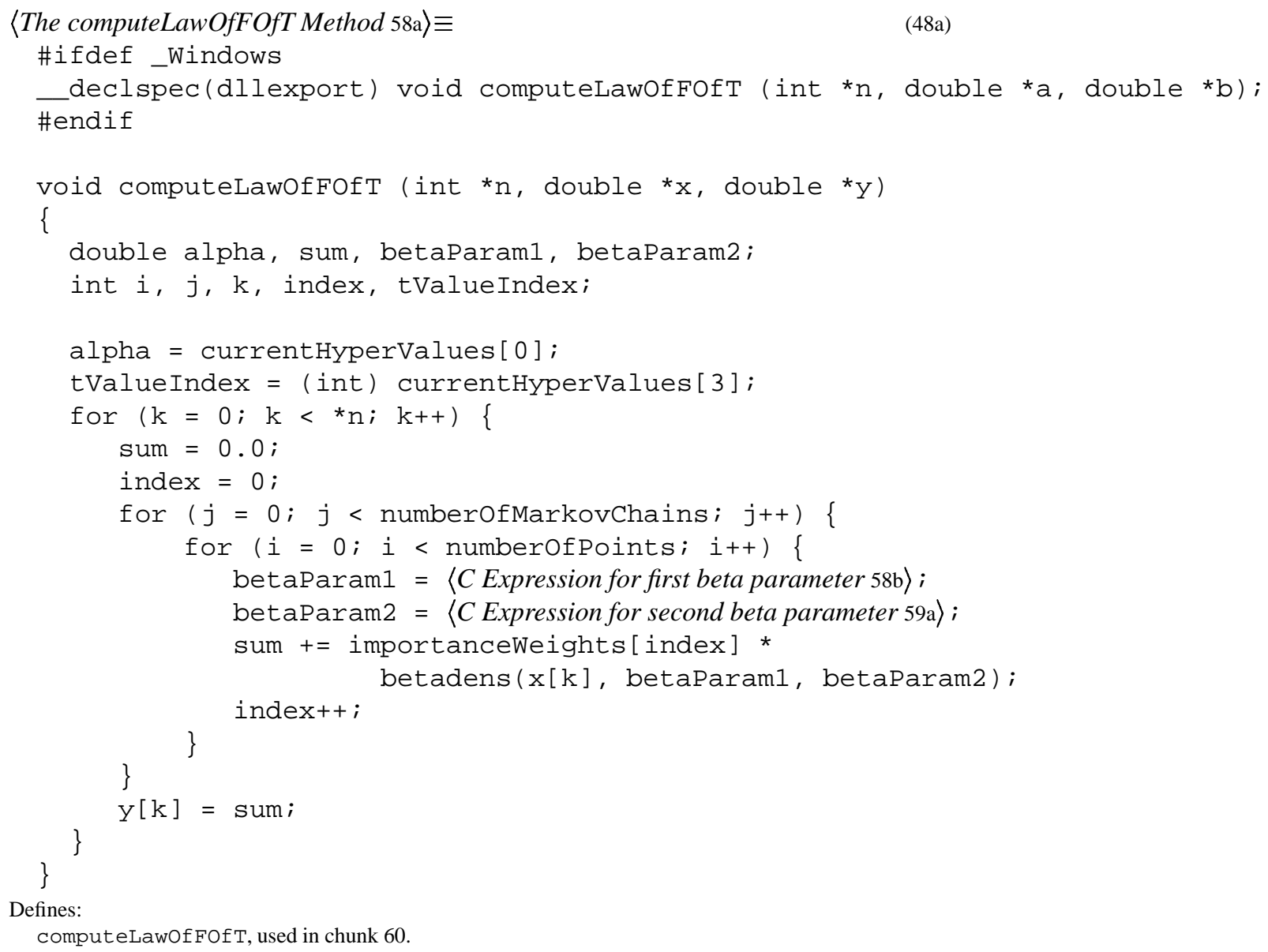

Here are the formulas for the two beta density parameters. The first one is of course

$$
\alpha_{\theta(g)}^{(\kappa)}(t)+\sum_{i=1}^{n} \delta_{X_{i}^{(g)}}(t)
$$

with the understanding that $\alpha$ indicates degree of concentration around the exponential family for theta.

The expression for the first beta parameter follows.

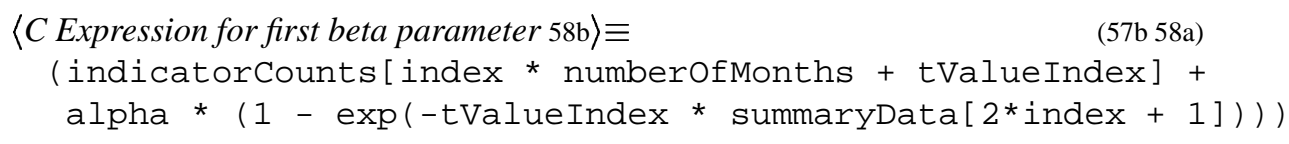


The second one is

$$
M_{\theta(g)}^{(\kappa)}+n-\alpha_{\theta^{(g)}}^{(\kappa)}(t)-\sum_{i=1}^{n} \delta_{X_{i}^{(g)}}(t)
$$

which in $\mathrm{C}$ is given by the snippet below.

\subsection{The C Equivalent of : compute-mean-of-fbar-of-t Method}

See : compute-mean-of-fbar-of-t method for slave-proto. This method basically calculates for several values of $t$, the quantity $E(S(t))$. The values of $t$ for which this is done is the same as the list of values used in the slider for $t$.

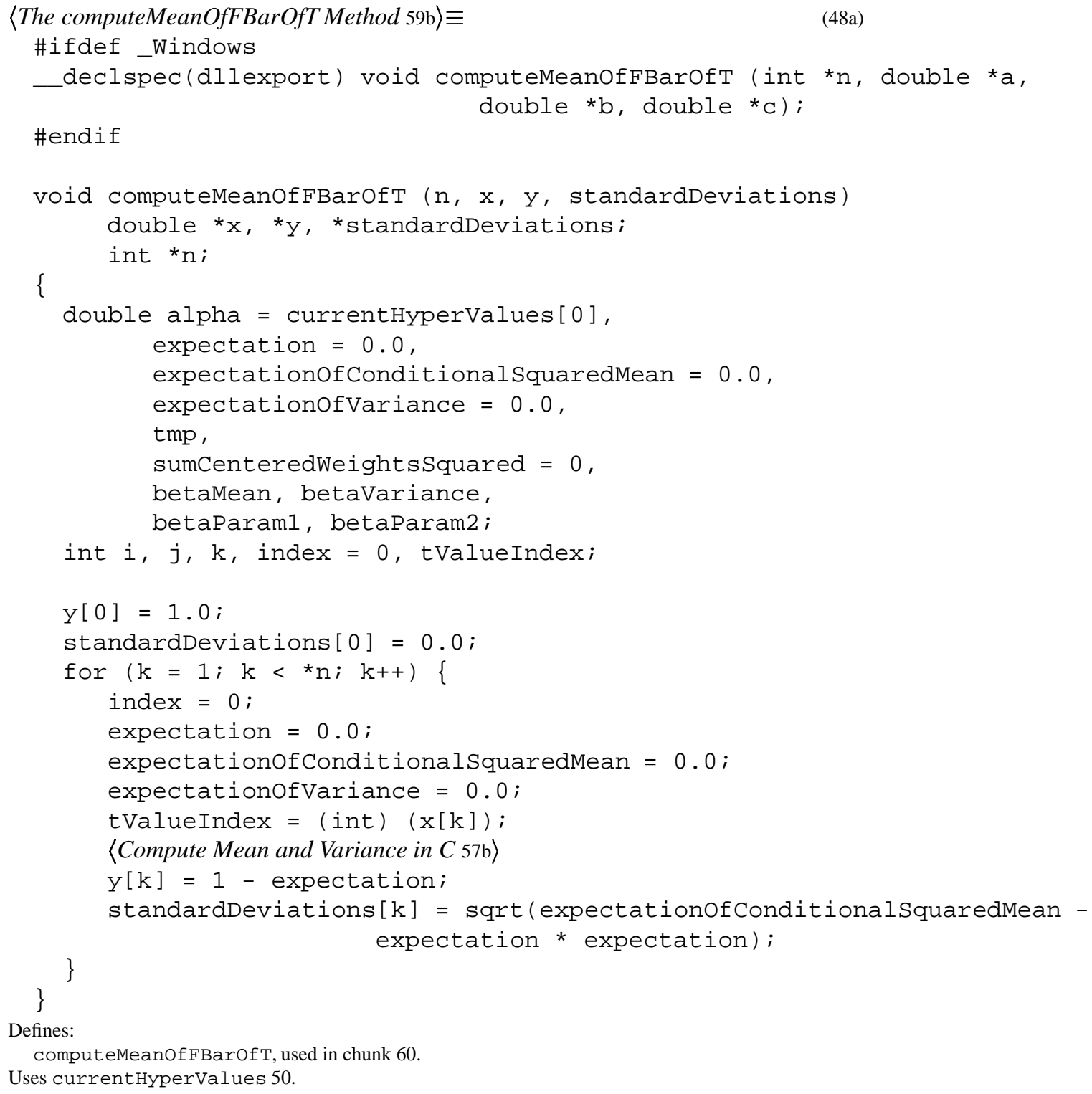




\subsection{The : consolidate-computation Method in C}

This method consolidates all computation by first computing importance weights, then computing all the statistics in one shot.

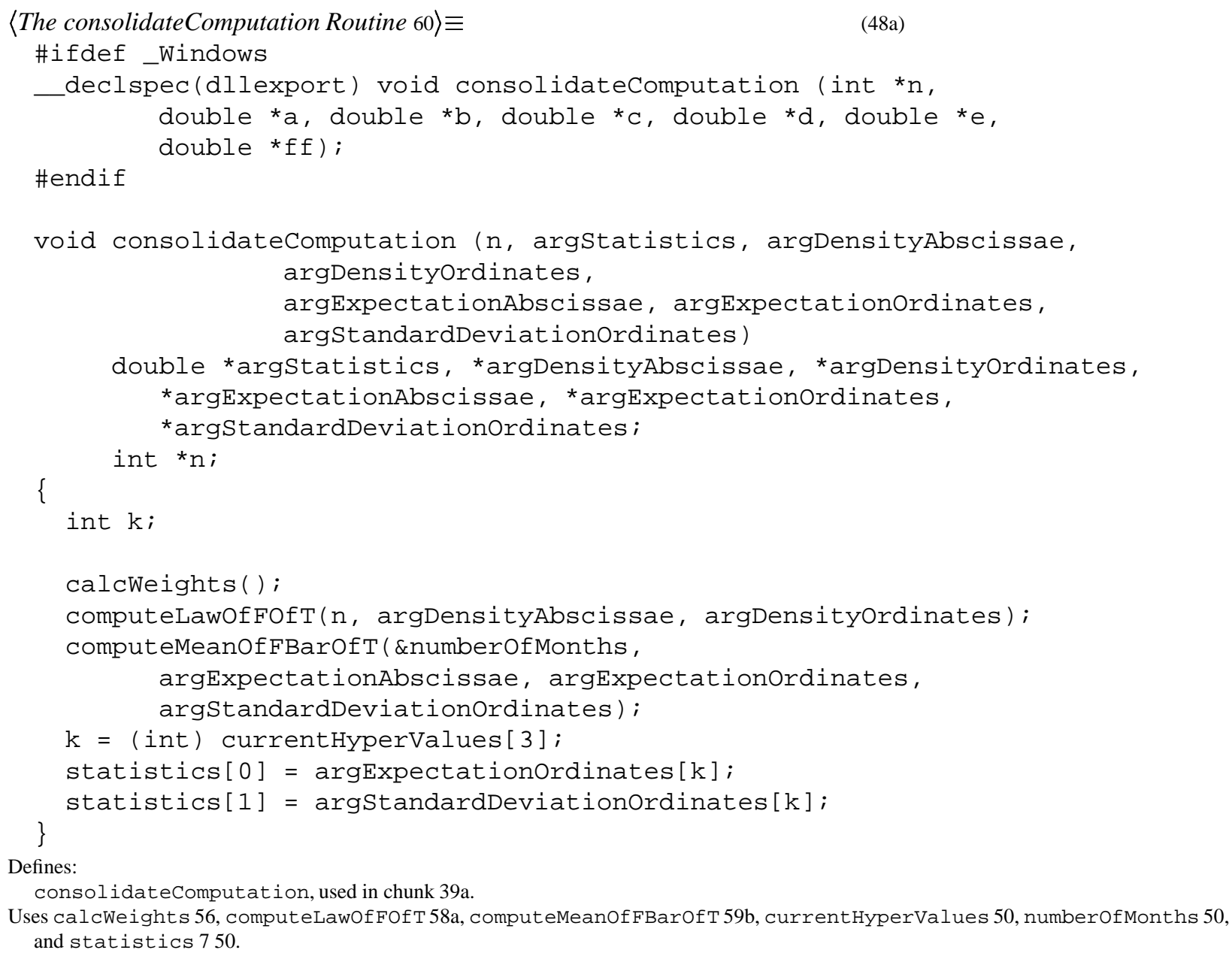


July 2, 1998

bsa.nw

\section{Installation Information}

We present some information on how to compile and use the software on various platforms. This is presented as a README file.

This file provides information on installing and using BSA (Bayesian Sensitivity Analysis) software. We expect any serious user of the software to read our paper that has now appeared in

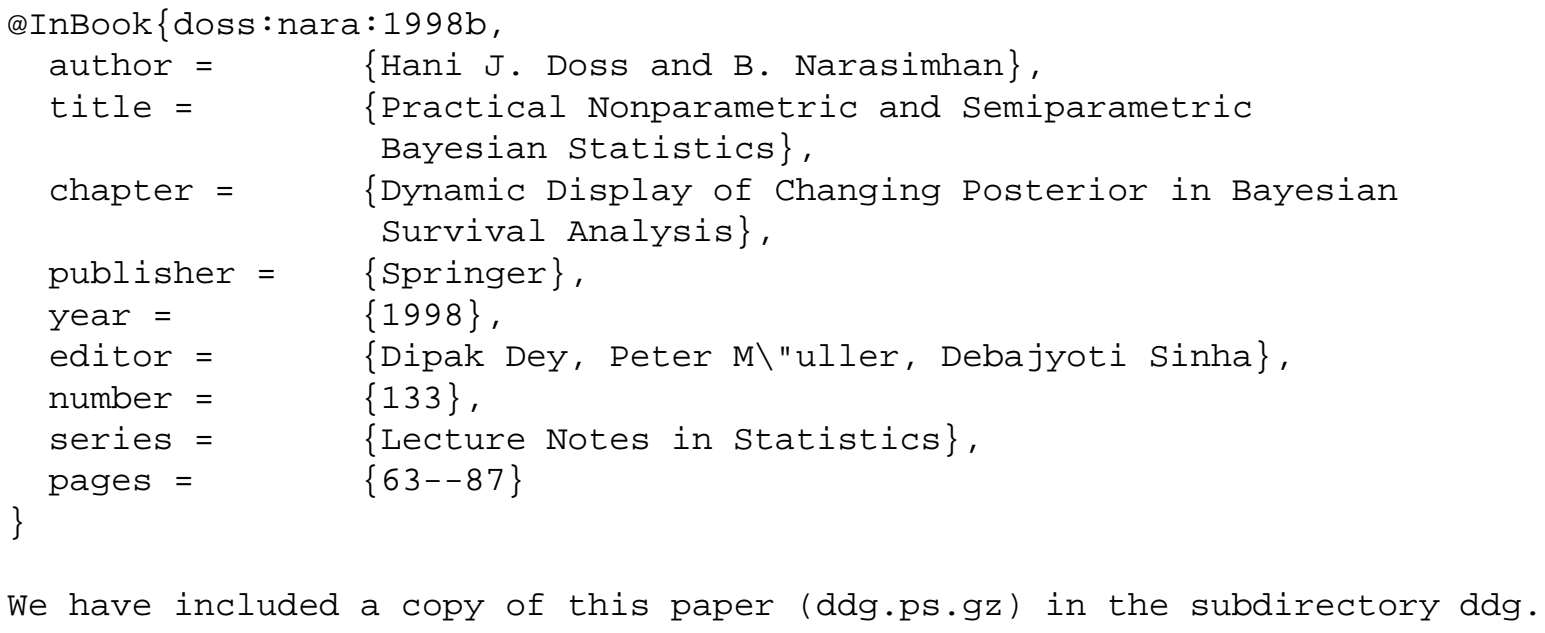

Requirements :
A) XLisp-Stat 3.53-5 or higher. Freely available from ftp://ftp.stat.umn.edu/pub/xlispstat/current
B) Windows or Unix. Windows includes 95 and NT.
(Mac version is in development.)
C) On Unix, you will also need a C compiler.

Step 1 .

You probably received the entire package as a compressed archive named bsa.tgz. On Unix, the contents of the archive may be extracted into a directory called bsa by executing the commands:

$$
\text { gunzip -c bsa.tgz| tar -xvf- }
$$

If you are using GNU tar, this can be done in one shot via: 


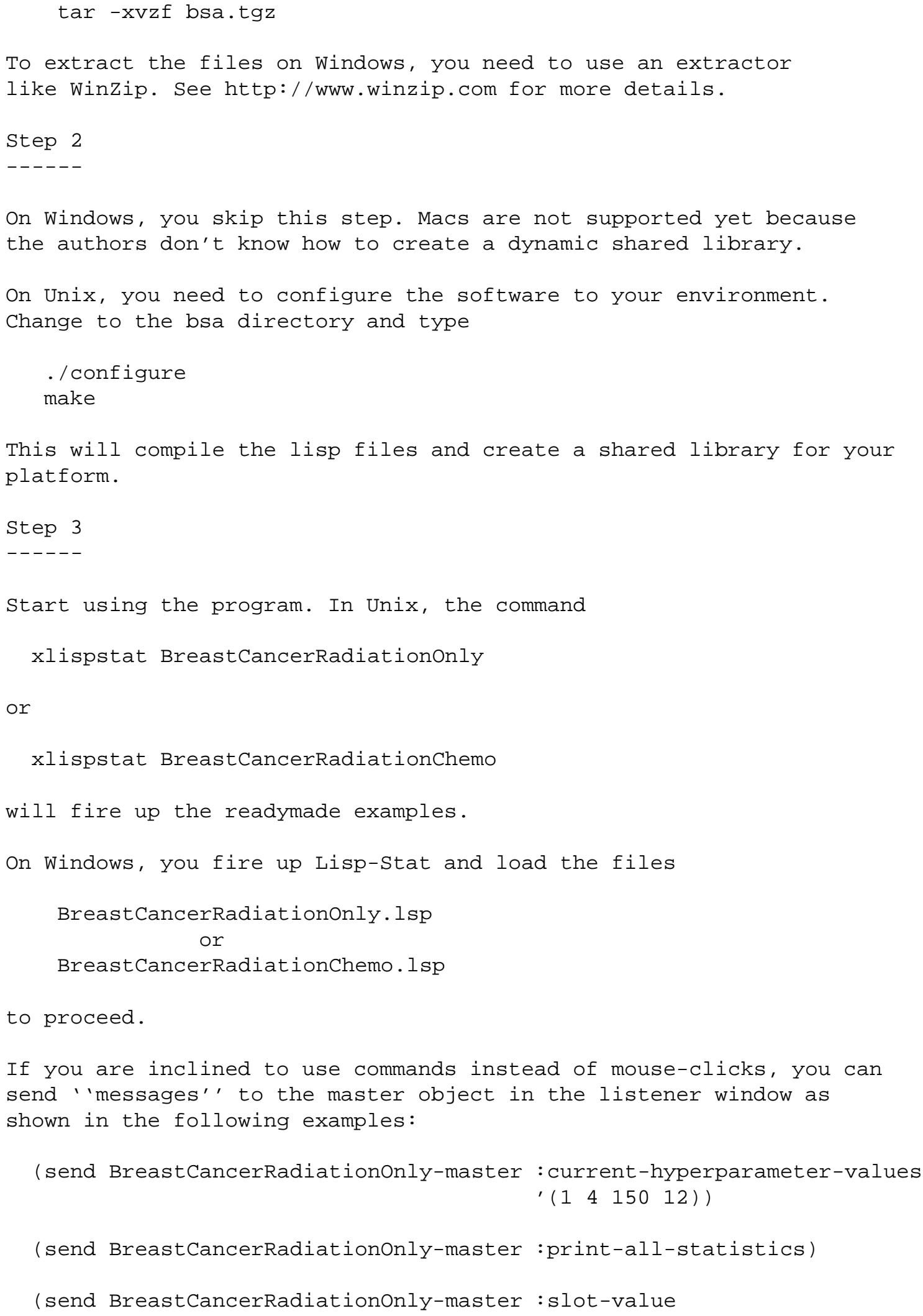


'bsa: : importance-weights)

Note how the master-objects names are related to the lisp file names and how internal slot-names have to be prefixed by the package name.

For dealing with a new problem, we provide a few points regarding the software. A number of inputs are required for running the program. These are discussed in detail in the literate program (bsa.ps) under the section titled 'Introduction.' For convenience we repeat the details here. This excerpted part is indented two spaces for easy reference.

First note that the software only does sensitivity analysis. No general facility is provided for generating observations from Markov chains. Indeed, since the range of models for which MCMC methods are applicable are large and such methods most likely involve problem-specific issues, it is our opinion that building such a supertool, if it is at all possible, is a non-trivial task. However, the Fortran program used in generating the output for our example is included along with this software and can be used for models similar to ours. Of course, any appropriate method may be used to generate the samples as long as the output is available in a form usable by our software. The requirements on the data that can be used with our software are spelt out below.

Corresponding to each Markov chain output, there must be two files with the extensions ".in" (input file) and ".out" (output file). For example, "mcl.in" and "mcl.out".

The input file must have the following structure. The first four items in the file can be anything, string or number, either on a single line or any conceivable combination of lines. The next three items *must* be the shape of the Gamma distribution on theta, the scale of the Gamma distribution on theta---the parametrization for shape $a$ and scale b is proportional to $x^{\wedge}\{a-1\} \exp (-\mathrm{bx})---a n d$ $M(R)$. The next three values values following these quantities can be anything, but the one following it should be the number of data points, that is, the number of sets or intervals. In the Fortran program we use -99 is used to denote infinity. Nothing else is read from the input file.

The output file must have the following structure for each data point generated by the Markov chain. The value of theta must be followed by the number of distinct values of the data points, which must be followed by a frequency table of the actual data value and the corresponding frequency. The layout of the values on lines does not matter as long as at least a single white space delimits values. If this structure is violated, errors will result. A peek at the data files included with this software will help the reader.

It is assumed that a proper installation of XLisp-Stat is available. 
For a new problem, you probably have several Markov chain output files although even one should work. (In the latter case, reweighting reduces to simple Importance Sampling.)

a) It is best to create a new directory for your problem and have your data files there. For example, the directory "BreastCancer" contains relevant data files for our Breast Cancer data.

b) The only files you actually need to run the program are:

1) Either one of bsa.fsl or bsa.lsp

2) Either one of utility.fsl or utility.lsp

3) Either one of call-by-reference.fsl or call-by-reference.lsp and

4) the shared library libbsa.so or libbsa.sl as the case may be. On Windows, instead of the shared library, we need the whole subdirectory "win".

Copy these files/directories to where you have the data files and work there.

5) The file new-problem.lsp.

Invoke Lisp-Stat and load the file named "new-problem.lsp".

The first time (and first time only), the following inputs will be needed.

Inputs

$------$

1) An indentifier for uniquely identifying the run. Use a meaningful name here. Let us assume this is BreastCancer (the default) in the discussion below.

2) The number of Markov chain outputs that you want to use for reweighting. Must be $>=1$, with 1 denoting straight Importance Sampling .

3) The names of the files containing output from Markov chains, *without the extensions*. The software will automatically tag on the extensions. in and. out when looking for files.

4) The number of points per chain to use in the dynamic reweighting. Thus if you specify 50 and have 8 chains, then 50 points from each of the eight chains $(=400)$ will be used.

5) An initial guess for maximizing the log-quasilikelihood which will provide an estimate of the constants of proportionality.

6) The range between which you want to vary the hyperparameters. If you use only one chain, then you *must* specify the range. Otherwise, the range will be a single point. If you specified many chains, the default settings for each hyperparameter will be the minimum and 
maximum values from values used in all Markov chains. The number of stops should be an odd number if you want to hit the middle of the interval.

7) The number of points to use in estimating the constants of proportionality. If you use all of the data, the estimation can take a while. It is almost always better to go with the default or less. (If you are really interested in using more points, then start off with 10, and use the estimates thus obtained to start your larger optimization. This will save you a lot of time.)

Once you specify this, the maximization will take place. This is a good point to go refill your coffee cup.

After the estimation, two files are created so that you are not bombarded with questions in subsequent explorations. For example,

$$
\text { BreastCancer. Isp (and) }
$$

BreastCancer. run

For repeating the exploration next time, you only need to load the file BreastCancer.lsp into XLisp-Stat. This will bypass all the inputs we discussed above except for the question about ranges. The file BreastCancer.run contains pre-processed information for faster loading and will be used when BreastCancer. lsp is loaded.

All files are text files and can be viewed with a text viewer.

Examples for Breast Cancer Data

The two files

BreastCancerRadiationonly.lsp (and)

BreastCancerRadiationChemo.lsp

and the corresponding run files are provided for experimentation. These exist in the main directory "bsa" itself and concern the dataset on two treatments described in the paper

Dynamic Display of Changing Posterior in Bayesian Survival Analysis by

Hani J. Doss and B. Narasimhan

By default, they use 50 points each and 8 Markov chains. We wish to note that an earlier version of the software was used to produce the results in the paper and subsequently a bug was found. This does not change any of the conclusions of the paper but the numbers shown in 


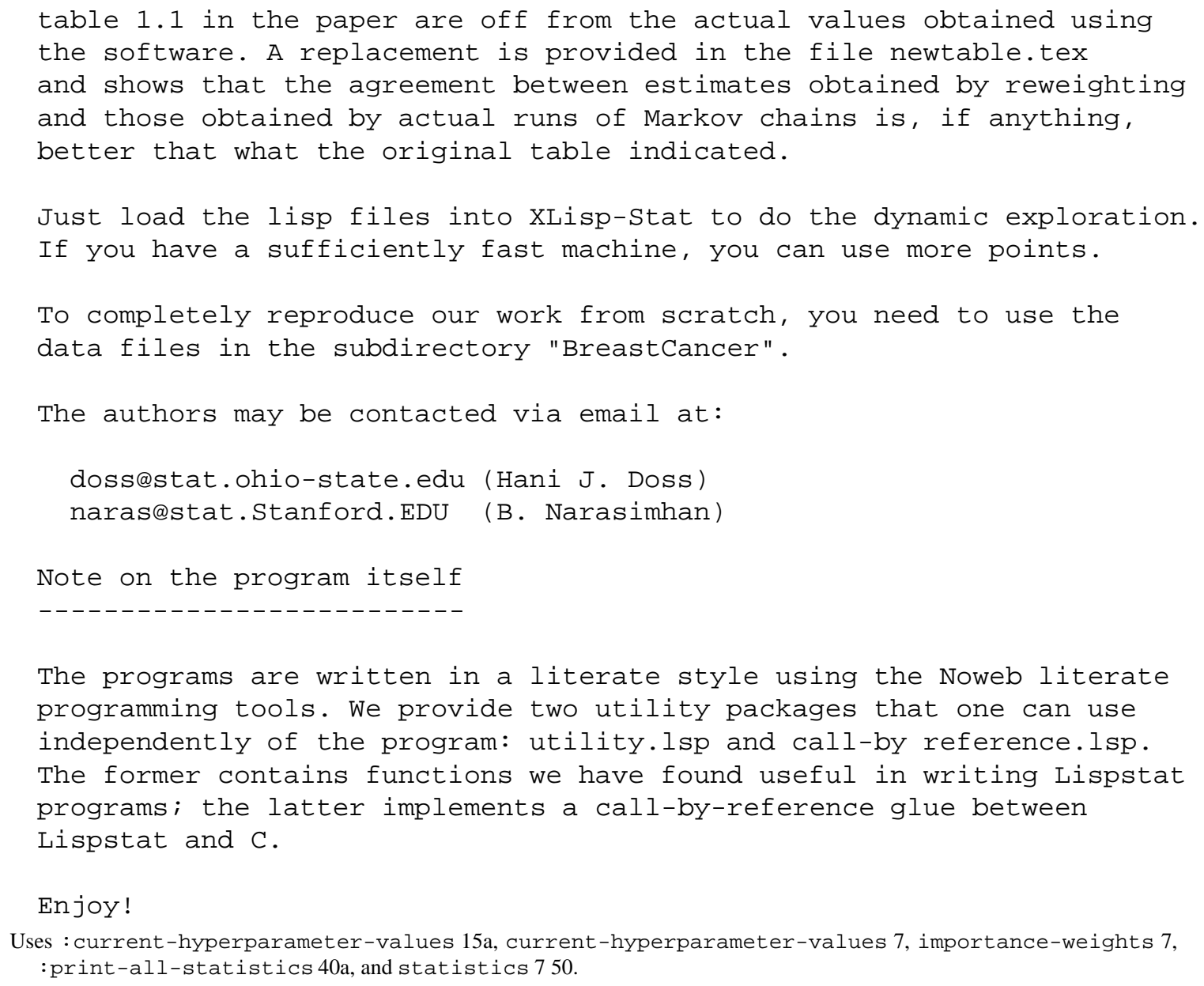




\section{Improvements needed}

- Make sure that the when a parameter hasn't changed then the hyperparameter range should not bomb. Right now, we've hardwired it. See the method : hyperparameter-ranges or follow it.

- Add ability for user to specify a sequence of values in the hyperparameter space along which snapshots may be saved. Thus reruns can be shown like animated weather maps on TV. Actually this can be done now as follows. Assume that hyperparameters-list is a list of hyperparameter points (that is, four-tuples), and master-object is the master object for the problem.

(dolist (hyperparameters hyperparameters-list)

(send master-object : current-hyperparameter-values hyperparameters))

- Clean up all the code that assumes that the time is an index varying from 0 to number-of-months. This has to be done carefully since it plays a role in many places.

\section{Acknowledgement}

This research was supported by Air Force Office of Scientific Research Grant F49620-94-1-0028.

We are indebted to Fred W. Huffer for the Fortran program dirichlet that comes with this software. All we did was modify it slightly to suit our data.

\section{Index of Code Chunks}

This list is generated automatically. The numeral is that of the first definition of the chunk.

$\langle * 5\rangle$

$\langle$ Address Initialization Routine 51 $\rangle$

$\langle$ Beta functions 49 $\rangle$

$\langle$ Bump starting index for next stretch 29e $\rangle$

$\langle C$ Copyright $47 \mathrm{~b}\rangle$

$\langle C$ Expression for first beta parameter 58b $\rangle$

$\langle C$ Expression for second beta parameter 59a

$\langle C$ Routines 48a

$\langle$ Compute Mean and Variance in C 57b

$\langle$ Copyright 4$\rangle$

$\langle$ Create lisp file for subsequent runs 37b

〈Create slaves of master object 24a〉

$\langle$ Defaults for Master 43a〉

$\langle$ Defaults for Slave 47a

$\langle$ Fill table entries with current frequency sum 29d $\rangle$

$\langle$ Find smallest month not less than $i$-th ordered $x$-val 29c $\rangle$

$\langle$ Get an identifier 32a $\rangle$

$\langle$ Get data file names 33〉

$\langle$ Get initial guess 34b $\rangle$

$\langle$ Get the number of Markov chains 32b

$\langle$ Get the number of points 34a

$\langle$ Global Variables 50

$\langle$ Handle situation when $x$-values run out before month values $30 \mathrm{~b}\rangle$

〈Header files 48b〉 
$\langle$ Make sliders with triples 25b

$\langle$ Make triples for sliders 25a

$\langle$ Methods for Master Prototype 10〉

$\langle$ Methods for Slave Prototype 43c

$\langle$ Pass data array addresses to C Routines 23a〉

$\langle$ Perform Reverse Logistic Regression 23c〉

〈Process Hyperparameter Ranges etc. 27d

〈Process the table containing info on Markov chains 28a)

$\langle$ Read frequency table and sort values 29b

$\langle$ Read in data files and set up data 34c $\rangle$

〈Read number of Markov chains 26b

$\langle$ Read number of months $27 \mathrm{c}\rangle$

$\langle$ Read number of points 27a〉

〈Read number of sets 27b〉

$\langle$ Read summary data, log mixture density, indicator counts 28b

$\langle$ README file 61 $\rangle$

$\langle$ Set up dialog and wait for user input 24b $\rangle$

$\langle$ Set up Hyperparameter-ranges and stops 20〉

$\langle$ Set up slot values for master object 18c $\rangle$

〈Show informative message 37c $\rangle$

〈Some final touches 25c

$\langle$ The calcWeights Routine 56

$\langle$ The computeLawOfFOfT Method 58a〉

$\langle$ The compute-log-hmix Routine 55〉

$\langle$ The computeMeanOfFBarOfT Method 59b〉

$\langle$ The computeStatistics Method 57a〉

$\langle$ The consolidateComputation Routine 60〉

$\langle$ The Density Routine 52〉

〈The hthetaOverHmix Routine 54b〉

$\langle$ The LogLikelihood Routine 54a〉

$\langle$ The LogProbability Routine 53〉

$\langle$ The Master :calc-weights Method 18a〉

$\langle$ The Master :close Method 42c〉

$\langle$ The Master :compute-log-hmix Method 17d

$\langle$ The Master: consolidate-computation Method 39a)

$\langle$ The Master :create-run-file Method 35b

$\langle$ The Master :current-hyperparameter-values Method 15a

〈The Master :effective-sample-size Method 39c〉

$\langle$ The Master :graphical-interface Method 31〉

$\langle$ The Master :hyperparameter-names Method 14c〉

$\langle$ The Master :hyperparameter-ranges Method 16b

$\langle$ The Master :hyperparameter-sliders Method 16c〉

$\langle$ The Master :hyperparameters-used-in-markov-chains Method 16a〉

$\langle$ The Master :identifier Method 12a

$\langle$ The Master :importance-weights Method 17b〉

$\langle$ The Master :indicator-counts Method 13c〉

〈The Master :initially-specified-hyperparameter-values Method 14b〉

〈The Master :isnew Method 18b〉

〈The Master :labelled-hyperparameter-values Method 40b〉

$\langle$ The Master :log-constants-of-proportionality Method 13d $\rangle$

$\langle$ The Master :loglik Method 17c〉 
$\langle$ The Master :log-mixture-density Method 17a

〈The Master :number-of-data-values Method 13a)

$\langle$ The Master :number-of-hyperparameters Method 15b〉

$\langle$ The Master :number-of-markov-chains Method 12b〉

$\langle$ The Master :number-of-months Method 12d〉

$\langle$ The Master : number-of-points Method 12c〉

$\langle$ The Master :print-all-statistics Method 40a〉

$\langle$ The Master :process-frequency-table Method 29a〉

$\langle$ The Master :process-run-file Method 26a〉

$\langle$ The Master Prototype 7〉

$\langle$ The Master :reset Method 39b〉

$\langle$ The Master :slaves Method 14a

〈The Master :statistics Method 41a〉

$\langle$ The Master :statistics-labels Method 41c〉

$\langle$ The Master : statistics-print-formats Method 41b

$\langle$ The Master : summary-data Method 13b

$\langle$ The Master :superimpose Method 42b

$\langle$ The Master :synchronize Method 38〉

$\langle$ The Master :toggle-timing Method 42a

$\langle$ The Slave :close Method 46b

$\langle$ The Slave :isnew Method 44a

$\langle$ The Slave :print-summary Method 46a〉

$\langle$ The Slave Prototype 43b

$\langle$ The Slave :redraw-background Method 44b

$\langle$ The Slave :redraw-statistics Method 45〉

$\langle$ Update frequency sum 30a

〈Write info on hyperparameters 36b

$\langle$ Write number of Markov chains, etc. 36a

$\langle$ Write summary data, log mixture density, indicator counts 37a

$\langle$ Write table of info on Markov chains 36c $\rangle$

\section{Index of Identifiers}

Here is a list of the identifiers used, and where they appear. Underlined entries indicate the place of definition. This index is generated automatically.]

betadens: $49,58 \mathrm{a}$

:calc-weights: $18 \mathrm{a}, 18 \mathrm{~b}$

calcWeights: $18 \mathrm{a}, \underline{56}, 60$

: close: 42c, 46b

computeLawOfFOft: $58 \mathrm{a}, 60$

compute-log-hmix: $17 \mathrm{~d}, 23 \mathrm{~b}$

computeLogHmix: $17 \underline{\mathrm{d}, \underline{55}}$

computeMeanoffBarofT: $\underline{59 \mathrm{~b}}, 60$

computestatistics: $\underline{57 a}$

: consolidate-computation: $38, \underline{39 a}$

consolidateComputation: $39 \mathrm{a}, \underline{60}$

copyright: $\underline{4}$

:current-hyperparameter-values: 15a, 25a, 25c, 38, 39b, 40b, 46a, 61

current-hyperparameter-values: 7, 15a, 25a, 25c, 38, 39b, 40b, 46a, 61

currentHyperValues: $\underline{50}$, 51, 54b, 57a, 58a, 59b, 60 


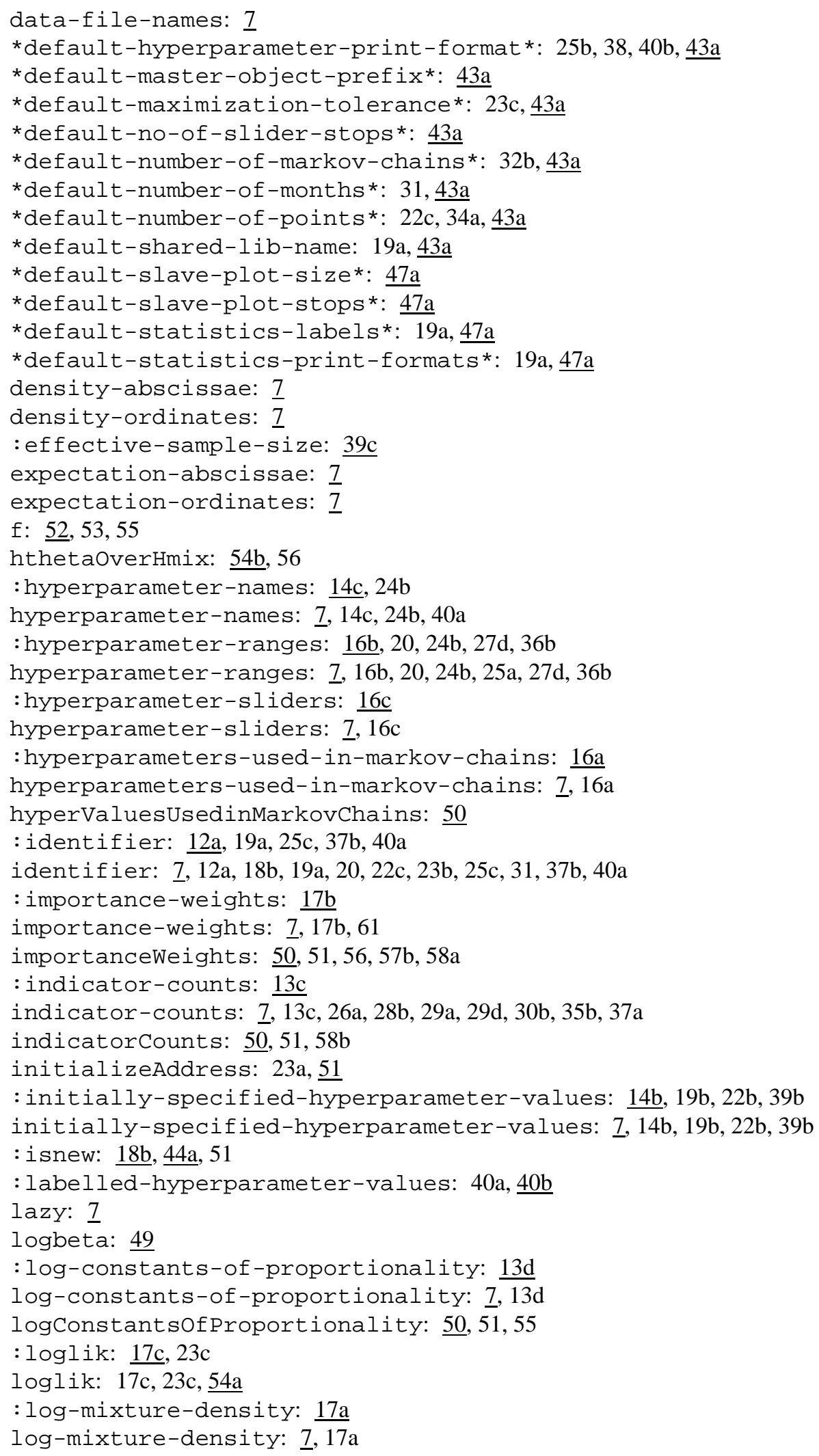


logMixtureDensity: $\underline{50}, 51,54 \mathrm{~b}, 55$

logp: $\underline{53}, 54 \mathrm{a}$

LOW_LOG_PROBABILITY: $\underline{50}, 53$

master-proto: 5, 7, 12a, 12b, 12c, 12d, 13a, 13b, 13c, 13d, 14a, 14b, 14c, 15a, 15b, 16a, 16b, 16c, 17a, 17b,

$17 \mathrm{c}, 17 \mathrm{~d}, 18 \mathrm{a}, 18 \mathrm{~b}, 26 \mathrm{a}, 29 \mathrm{a}, 31,35 \mathrm{~b}, 37 \mathrm{~b}, 38,39 \mathrm{a}, 39 \mathrm{~b}, 39 \mathrm{c}, 40 \mathrm{a}, 40 \mathrm{~b}, 41 \mathrm{a}, 41 \mathrm{~b}, 41 \mathrm{c}, 42 \mathrm{a}, 42 \mathrm{~b}, 42 \mathrm{c}$

mygamma: $\underline{49}, 52,54 \mathrm{~b}$

: number-of-data-values: $13 \mathrm{a}$

number-of-data-values: $1,13 \mathrm{a}$

numberOfDataValues: $\underline{50}, 51,59 \mathrm{a}$

: number-of-hyperparameters: 15a, 15b, 25a, 25c, 40b

: number-of-markov-chains: $12 \mathrm{~b}, 19 \mathrm{a}$

number-of-markov-chains: 7, 12b, 16a, 17b, 18b, 19a, 31, 32b, 43a

numberOfMarkovChains: $\underline{50}, 51,53,54 \mathrm{a}, 55,56,57 \mathrm{~b}, 58 \mathrm{a}$

: number-of-months: $12 \mathrm{~d}, 22 \mathrm{a}$

number-of-months: $\underline{7}, 12 \mathrm{~d}, 13 \mathrm{c}, 22 \mathrm{a}, 29 \mathrm{a}, 29 \mathrm{c}, 30 \mathrm{~b}, 31,43 \mathrm{a}$

numberOfMonths: $\underline{50}, 51,58 \mathrm{~b}, 60$

: number-of-points: $12 \mathrm{c}, 19 \mathrm{a}$

number-of-points: 7, 12c, 17b, 18b, 19a, 22c, 26a, 27a, 31, 34a, 43a

numberOfPoints: $\underline{50}, 51,55,56,57 \mathrm{~b}, 58 \mathrm{a}$

numberOfPointsForConstants: 50, 51, 54a

number-of-slider-stops: $\underline{7}$

:print-all-statistics: $24 \mathrm{~b}, \underline{40 \mathrm{a}}, 61$

:print-summary: 40a, $\underline{46 a}$

:process-frequency-table: $\underline{29 a}, 34 c$

:process-run-file: 19a, 26a, $\underline{28 b}$

:redraw-background: $\underline{44 \mathrm{~b}}$

:redraw-statistics: $44 \mathrm{~b}, \underline{45}$

: reset: $24 \mathrm{~b}, \underline{39 \mathrm{~b}}$

shared-library: 7

slave-proto: $24 \mathrm{a}, \underline{43 \mathrm{~b}}, 44 \mathrm{a}, 44 \mathrm{~b}, 45,46 \mathrm{a}, 46 \mathrm{~b}$

:slaves: $14 \mathrm{a}$

slaves: $\underline{7}, 14 \mathrm{a}, 38$

standard-deviation-ordinates: 7

:statistics: $\underline{41 \mathrm{a}}, 41 \mathrm{~b}, 41 \mathrm{c}, 44 \mathrm{a}, 45$

statistics: 7, 19a, 24b, 39a, 40a, 41a, 41b, 41c, 44a, 44b, 45, 47a, 50, 51, 56, 60, 61

:statistics-labels: $41 \mathrm{c}, 45$

statistics-labels: $\underline{7}, 19 \mathrm{a}, 41 \mathrm{c}, 45,47 \mathrm{a}$

:statistics-print-formats: $\underline{41 \mathrm{~b}}, 44 \mathrm{a}, 45$

statistics-print-formats: 1 , 19a, 41b, 44a, 45, 47a

: summary-data: $\underline{13 b}$

summary-data: $\underline{7}, 13 \mathrm{~b}, 26 \mathrm{a}, 28 \mathrm{~b}, 31,34 \mathrm{c}, 35 \mathrm{a}, 35 \mathrm{~b}, 37 \mathrm{a}$

summaryData: $\underline{50}, 51,52,54 \mathrm{~b}, 58 \mathrm{~b}$

: superimpose: $\underline{42 \mathrm{~b}}$

superimpose: $1,42 \mathrm{~b}$

: synchronize: $15 \mathrm{a}, 25 \mathrm{c}, \underline{38}$

timing: $\underline{7}, 24 \mathrm{~b}, 42 \mathrm{a}$

:toggle-timing: 24b, $\underline{42 \mathrm{a}}$

work-space: $\underline{7}$ 
July 2, 1998

bsa.nw

\section{References}

[1] Hani J. Doss and B. Narasimhan. Bayesian Poisson regression: Sensitivity analysis through dynamic graphics. Technical report, Penn State Erie, The Behrend College, 1994.

[2] Hani J. Doss and B. Narasimhan. Dynamic display of changing posterior in Bayesian survival analysis. In Practical Nonparametric and Semiparametric Bayesian Statistics, D. Dey, P. Müller, and D. Sinha, eds, 1998. Springer-Verlag, N. Y.

[3] C. J. Geyer. Estimating normalizing constants and reweighting mixtures in Markov chain Monte Carlo. Technical report, School of Statistics, University of Minnesota, 1994.

[4] Donald E. Knuth. Literate Programming. Center for Study of Language and Information, Stanford University, 1992.

[5] A. Kong, J. S. Liu, and W. H. Wong. Sequential imputations and Bayesian missing data problems. J. Amer. Statist. Assoc., 1994.

[6] Christopher Lee. Literate programming—propaganda and tools. World Wide Web, 1994. Available from http: //www.ius.cs.cmu.edu/help/Programming/literate.html.

[7] Norman Ramsey. Literate programming simplified. IEEE Software, 1994.

[8] Norman Ramsey. The Noweb Hacker's Guide, 1994. Manual for Noweb.

[9] Norman Ramsey. The noweb home page. World Wide Web, 1995. On the Web at http://www.cs . virginia.edu/〜nr/noweb/.

[10] Luke Tierney. LISP-STAT: An Object-Oriented Environment for Statistical Computing and Dynamic Graphics. John Wiley \& Sons, 1990. 